\title{
SCHATTEN-VON NEUMANN PROPERTIES OF BILINEAR HANKEL FORMS OF HIGHER WEIGHTS
}

\author{
MARCUS SUNDHÄLL*
}

\begin{abstract}
Hankel forms of higher weights, on weighted Bergman spaces in the unit ball of $\mathrm{C}^{d}$, were introduced by Peetre. Each Hankel form corresponds to a vector-valued holomorphic function, called the symbol of the form. In this paper we characterize bounded, compact and Schatten-von Neumann $\mathscr{S}_{p}$ class $(2 \leq p<\infty)$ Hankel forms in terms of the membership of the symbols in certain Besov spaces.
\end{abstract}

\section{Introduction and Main Results}

\subsection{Introduction}

Hankel operators on the unit disc have been studied extensively and have found many applications, see [13], [22] and [8]. One of the central problems is to study the characterization of their Schatten-von Neumann properties. We recall briefly the definition of Hankel operators on a Hardy space on the unit disc. Consider the Hardy space $H^{2}(T) \subset L^{2}(T)$ of holomorphic functions, where $T=\{z \in \mathrm{C}:|z|=1\}$. Let $P: L^{2}(T) \rightarrow H^{2}(T)$ be the Szegó projection. The Hankel operator $\tilde{H}_{f}$ with holomorphic symbol $f$ is defined by $\tilde{H}_{f} g=(I-P)(\bar{f} g), g \in H^{2}(T)$. It can also be viewed (up to a rank one operator) as a bilinear form $H_{f}$ on $H^{2}(T)$, namely

$$
H_{f}\left(g_{1}, g_{2}\right)=\int_{\partial D} \overline{f(z)} g_{1}(z) g_{2}(z) d \sigma(z) .
$$

Their Schatten-von Neumann properties were studied first by Peller, see [14]. It is proved there that $H_{f}$ is of Schatten-von Neumann class if and only if $f$ is in a certain Besov space. The corresponding problem for Hankel forms on a Bergman space has been studied in [8] and [18]. It was realized later that the Hilbert-Schmidt Hankel forms on a weighted Bergman space can be viewed as the first irreducible component in the irreducible decomposition of the tensor

* The author is financially supported by Örebro University.

Received September 17, 2004; in revised form February 28, 2005. 
product of two copies of the Bergman spaces, and subsequently Janson and Peetre [7] introduced the Hankel forms of higher weights on Bergman spaces on the unit disc; see also [19] where multilinear Hankel forms are studied.

A natural problem is to consider Hankel forms on the unit ball in $\mathrm{C}^{d}$. In [11] Peetre introduced Hankel forms on the unit ball. As in the case of the unit disc the spaces of Hankel forms of higher weights are explicit characterization of irreducible components in the tensor product of Bergman spaces under the Möbius group, see [7], [11] and [15]. However their Schatten-von Neumann properties have not been studied so far. In this paper we will address this problem.

The Hilbert and Banach spaces of symbols appearing in this paper are closely related to the quotients of function modules studied in [4], and the expansion of the reproducing kernels of some similar spaces have been studied in [6]. It is interesting to consider those problems in our context.

Part of this paper is from my licentiate thesis under the supervision of my advisor Genkai Zhang. I would like to thank him for many fruitful discussions. I am also grateful for all the encouragement and help from my supervisor Yang Liu. I thank also the referee for his/her comments, remarks and for pointing out certain incomplete arguments. After the first manuscript was submitted we have established a necessary and sufficient condition for the membership of Hankel forms in Schatten-von Neumann class $\mathscr{S}_{p}, 2<p<\infty$, by studying the Bergman type projections (see Section 7 and Section 8).

The paper is arranged in the following manner. In Section 1 we introduce the Hankel forms and state the main results in the form of three theorems. Section 2 consists of preliminary results. Section 3 is devoted to certain Banach spaces of vector-valued holomorphic functions. Section 4 gives an equivalent description for certain Besov spaces. The proofs of Theorem 1.1(a) and Theorem 1.1(b) are given in Section 5 and Section 6 respectively. The proof of Theorem 1.2 is given in Section 6. In Section 7 we prove some $L^{P}$-boundedness properties of certain Bergman type projections, which are used in Section 8 to prove Theorem 1.3.

\subsection{Notation}

Let $H_{1}$ and $H_{2}$ be Hilbert spaces and let $T: H_{1} \rightarrow H_{2}$ be a linear operator. Define the singular numbers $s_{n}(T)=\inf \{\|T-K\|: \operatorname{rank}(K) \leq n\}, n \geq 0$. If $T$ is compact, these singular numbers are equal to the eigenvalues of $|T|=$ $\left(T^{*} T\right)^{1 / 2}$. We denote by $\mathscr{S}_{p}$ the ideal of operators for which $\left\{s_{n}(T)\right\}_{n \geq 0} \in l^{p}$, $0<p \leq \infty$, see [21]. We remark that $\mathscr{S}_{\infty}$ is the class of bounded operators. (The compact operators correspond to $c_{0}$, not to $l^{\infty}$.)

Let $d m$ denote the Lebesgue measure on the unit ball $\mathrm{B} \subset \mathrm{C}^{d}$ and let $d \iota(z)$ be the measure $\left(1-|z|^{2}\right)^{-d-1} d m(z)$. For $d<v<\infty$ let $d \iota_{v}(z)$ be the measure 
$c_{v}\left(1-|z|^{2}\right)^{v} d \iota(z)$, where $c_{v}$ is chosen such that

$$
\int_{\mathrm{B}} d \iota_{v}(z)=1
$$

i.e., $c_{v}=\Gamma(v) /\left(\pi^{d} \Gamma(v-d)\right)$. The closed subspace of all holomorphic functions in $L^{2}\left(d \iota_{v}\right)$ is denoted by $L_{a}^{2}\left(d \iota_{v}\right)$ and is called a weighted Bergman space. Note that the space $L_{a}^{2}\left(d \iota_{v}\right)$ has a reproducing kernel $K_{z}(w)=(1-\langle w, z\rangle)^{-v}$, that is,

(1) $f(z)=\left\langle f, K_{z}\right\rangle_{\nu}=\int_{\mathrm{B}} f(w) \overline{K_{z}(w)} d \iota_{v}(w), \quad f \in L_{a}^{2}\left(d \iota_{v}\right), \quad z \in \mathrm{B}$.

Denote by $B(z, w)$ the Bergman operator on $V=\mathrm{C}^{d}$ as in [10], namely

$$
B(z, w)=(1-\langle z, w\rangle)\left(I-z \otimes w^{*}\right),
$$

where $z \otimes w^{*}$ stands for the rank one operator given by $\left(z \otimes w^{*}\right)(v)=\langle v, w\rangle z$. Viewed as a matrix acting on column vectors it is

$$
B(z, w)=(1-\langle z, w\rangle)\left(I-z \bar{w}^{t}\right),
$$

where $w^{t}$ is the transpose of $w . B(z, w)$ is holomorphic in $z$ and antiholomorphic in $w$.

The Bergman metric at $z \in \mathrm{B}$, when we identify the tangent space with $V$, is $\left\langle B(z, z)^{-1} u, v\right\rangle$ for $u, v \in V$. We note that

$$
B(z, w)^{-1}=(1-\langle z, w\rangle)^{-2}\left((1-\langle z, w\rangle) I+z \otimes w^{*}\right) .
$$

Let $B^{t}(z, w)$ denote the dual of $B(z, w)$ acting on the dual space $V^{\prime}$ of $V$. When acting on a vector $v^{\prime} \in V^{\prime}$ it is

$$
B^{t}(z, w) v^{\prime}=(1-\langle z, w\rangle) v^{\prime}\left(I-z \bar{w}^{t}\right) .
$$

Actually we may identify $B^{t}(z, w)$ with $(1-\langle z, w\rangle)\left(I-\bar{w} z^{t}\right)$.

For a non-negative integer $s$, let $\otimes^{s} V^{\prime}$ be the tensor product of $s$ factors $V^{\prime}$ and let $\otimes^{0} V^{\prime}=\mathrm{C}$. The space $\otimes^{s} V^{\prime}$ is equipped with a natural Hermitian inner product induced by that of $V^{\prime}$, so that

$$
\left\langle v_{1} \otimes \cdots \otimes v_{s}, w_{1} \otimes \cdots \otimes w_{s}\right\rangle=\prod_{j=1}^{s}\left\langle v_{j}, w_{j}\right\rangle
$$

where $v_{j}, w_{j} \in V^{\prime}, j=1, \ldots, s$. 
Let $\left\{u_{1}, \ldots, u_{d}\right\} \subset V^{\prime}$. Denote by $u_{1}^{i_{1}} \odot u_{2}^{i_{2}} \odot \cdots \odot u_{d}^{i_{d}}$ the sum

$$
\frac{i_{1} ! \cdots i_{d} !}{s !} \sum_{\pi \in S} \pi(\underbrace{u_{1} \otimes \cdots \otimes u_{1}}_{i_{1} \text { factors }} \otimes \cdots \otimes \underbrace{u_{d} \otimes \cdots \otimes u_{d}}_{i_{d} \text { factors }})
$$

where $i_{1}+\cdots+i_{d}=s, S=S_{s} /\left(S_{i_{1}} \times \cdots \times S_{i_{d}}\right), S_{s}$ is the permutation group acting on the tensor by permutating the factors in the tensor and $S_{i_{1}}, \ldots, S_{i_{d}}$ are the subgroups permutating the first $i_{1}$, the second $i_{2}, \ldots$, the last $i_{d}$ elements respectively.

Let $\left\{e_{1}, \ldots, e_{d}\right\}$ be a basis for $V^{\prime}$. Denote by $\odot^{s} V^{\prime}$ the subspace of symmetric tensors of length $s$

$$
\left\{\sum_{i_{1}+\cdots+i_{d}=s} v_{i} e_{1}^{i_{1}} \odot e_{2}^{i_{2}} \odot \cdots \odot e_{d}^{i_{d}}: i=\left(i_{1}, \ldots, i_{d}\right) \in \mathrm{N}^{d}, v_{i} \in \mathrm{C}\right\} .
$$

Also, denote by $\otimes^{s} B^{t}(z, z)$ the operator on $\otimes^{s} V^{\prime}$ induced by the action of $B^{t}(z, z)$ on $V^{\prime}$, where $\otimes^{0} B^{t}(z, z)=I$.

\subsection{Hankel forms and main results}

The Transvectant $\mathscr{T}_{s}$ on $L_{a}^{2}\left(d \iota_{v}\right) \otimes L_{a}^{2}\left(d \iota_{v}\right)$ (introduced in [11], see also [12] and [15]) is defined by

$$
\mathscr{T}_{s}(f, g)(z)=\sum_{k=0}^{s}\left(\begin{array}{l}
s \\
k
\end{array}\right)(-1)^{s-k} \frac{\partial^{k} f(z) \odot \partial^{s-k} g(z)}{(v)_{k}(v)_{s-k}}
$$

where

$$
\partial^{s} f(z)=\sum_{j_{1} \ldots j_{s}=1}^{d} \partial_{j_{1}} \cdots \partial_{j_{s}} f(z) d z_{j_{1}} \otimes \cdots \otimes d z_{j_{s}} \in \odot^{s} V^{\prime}
$$

and $(v)_{k}=v(v+1) \cdots(v+k-1),(v)_{0}=1$, is the Pochammer symbol.

The Hankel bilinear form $H_{F}^{s}$ on $L_{a}^{2}\left(d \iota_{v}\right) \otimes L_{a}^{2}\left(d \iota_{v}\right)$ is defined by

$$
H_{F}^{s}(f, g)=\int_{\mathrm{B}}\left\langle\otimes^{s} B^{t}(z, z) \mathscr{T}_{s}(f, g)(z), F(z)\right\rangle d \iota_{2 v}(z)
$$

where $F: \mathrm{B} \rightarrow \odot^{s} V^{\prime}$ is holomorphic. We call $F$ the symbol of the corresponding Hankel form. We remark that

$$
H_{F}^{0}(f, g)=\int_{\mathrm{B}} f(z) g(z) \overline{F(z)} d \iota_{2 v}(z) .
$$

This is the classical Hankel form studied in [8]. 
With the form $H_{F}^{s}$ one can associate the operator $A_{F}^{s}$ defined by

$$
H_{F}^{s}(f, g)=\left\langle f, A_{F}^{s} g\right\rangle_{v}
$$

as in [8]. Notice that $A_{F}^{s}$ is an anti-linear operator on $L_{a}^{2}\left(d \iota_{v}\right)$. To get a linear operator one combines $A_{F}^{s}$ with a conjugation, i.e., one instead considers the operator $\bar{A}_{F}^{s}: g \rightarrow \overline{A_{F}^{s} g}$. We say that $H_{F}^{s}$ is of Schatten-von Neumann class $\mathscr{S}_{p}$, for $0<p<\infty$, if and only if $\bar{A}_{F}^{s}: L_{a}^{2}\left(d \iota_{v}\right) \rightarrow \overline{L^{2}\left(d \iota_{v}\right)}$ is of class $\mathscr{S}_{p}$.

Finally we present the main results, of this paper, in the form of three theorems where we let $s$ be a non-negative integer.

THeORem 1.1. Let $F: \mathrm{B} \rightarrow \odot^{s} V^{\prime}$ be a holomorphic function.

(a) $H_{F}^{s}$ is bounded if and only if

$$
\sup _{z \in \mathrm{B}}\left\langle\left(1-|z|^{2}\right)^{2 v} \otimes^{s} B^{t}(z, z) F(z), F(z)\right\rangle<+\infty,
$$

(b) $H_{F}^{s}$ is compact if and only if

$$
\left\langle\left(1-|z|^{2}\right)^{2 v} \otimes^{s} B^{t}(z, z) F(z), F(z)\right\rangle \rightarrow 0 \quad \text { as } \quad|z| \nearrow 1 .
$$

THEOREM 1.2. $H_{F}^{s}$ is of Hilbert-Schmidt class $\mathscr{S}_{2}$ if and only if

$$
\int_{B}\left\langle\left(1-|z|^{2}\right)^{2 v} \otimes^{s} B^{t}(z, z) F(z), F(z)\right\rangle d \iota(z)<+\infty .
$$

Theorem 1.3. $H_{F}^{s}$ is of class $\mathscr{S}_{p}$, for $2<p<\infty$, if and only if

$$
\int_{B}\left\langle\left(1-|z|^{2}\right)^{2 v} \otimes^{s} B^{t}(z, z) F(z),\left.F(z)\right|^{p / 2} d \iota(z)<+\infty .\right.
$$

\section{Preliminaries}

2.1. $G=\operatorname{Aut}(\mathrm{B}):$ The automorphisms of $\mathrm{B}$

We shall need some results on the group $G=\operatorname{Aut}(\mathrm{B})$ of biholomorphic mappings of $\mathrm{B}$.

Let $P_{z}$ be the orthogonal projection of $\mathrm{C}^{d}$ into $\mathrm{C} z$ and let $Q_{z}=I-P_{z}$. Put $s_{z}=\left(1-|z|^{2}\right)^{1 / 2}$ and define a linear fractional mapping $\varphi_{z}$ on B by

$$
\varphi_{z}(w)=\frac{z-P_{z} w-s_{z} Q_{z} w}{1-\langle w, z\rangle} .
$$


If $g \in G$ and $g(z)=0$ then there is a unique unitary operator $U: \mathrm{C}^{d} \rightarrow \mathrm{C}^{d}$ such that

$$
g=U \varphi_{z}
$$

Sometimes $g(z)$ will be written as $g z$. Define the complex Jacobian $J_{g}$ by $J_{g}(w)=\operatorname{det}\left(g^{\prime}(w)\right)$. Then we have $J_{g}(w)=\operatorname{det} U \cdot J_{\varphi_{z}}(w)$. Lemma 2.1 gives the differential of the Möbius transformations. It can be proved by similar computations as in the proof of Theorem 2.2.2 in [20].

Lemma 2.1. Let $\varphi_{z}$ be the linear fractional mapping (8) on $\mathrm{B}$. Then

$$
\varphi_{z}^{\prime}(w)=\frac{-s_{z}^{2} P_{z}-s_{z} Q_{z}+s_{z}\left(\langle w, z\rangle-w \otimes z^{*}\right)}{(1-\langle w, z\rangle)^{2}} .
$$

By computating the determinant of $\varphi_{z}^{\prime}(w)$ we get the next proposition. It is a refinement of Theorem 2.2.6 in [20], which we state as a corollary.

Proposition 2.2. Let $\varphi_{z}$ be the linear fractional mapping (8) on $\mathrm{B}$. Then

$$
J_{\varphi_{z}}(w)=(-1)^{d}\left(\frac{s_{z}}{1-\langle w, z\rangle}\right)^{d+1} .
$$

Corollary 2.3. Let $g \in G$. Then the real Jacobian $J_{\mathrm{R}, g}$ of $g$ is

$$
J_{\mathrm{R}, g}(w)=\left|J_{g}(w)\right|^{2}=\left(\frac{1-|z|^{2}}{|1-\langle w, z\rangle|^{2}}\right)^{d+1} .
$$

We need also the Forelli-Rudin estimate (see Proposition 1.4.10 in [20]).

Lemma 2.4. Let $\gamma>\alpha>d$. Then

$$
\int_{\mathbf{B}} \frac{\left(1-|w|^{2}\right)^{\alpha}}{|1-\langle z, w\rangle|^{\gamma}} d \iota(w) \leq C\left(1-|z|^{2}\right)^{-(\gamma-\alpha)} .
$$

\subsection{Some elementary properties of the Bergman operator}

Let $g \in G$. Combining Proposition IX.1.1 with Proposition IX.2.6 in [3] we get

$$
B(z, w)^{-1}=(d g(w))^{*} B(g z, g w)^{-1} d g(z) .
$$

This yields

$$
B^{t}(g z, g w)=\left(d g(w)^{t}\right)^{*} B^{t}(z, w) d g(z)^{t} .
$$


Now we consider another property of the Bergman operator. It holds that

$$
B^{t}(z, z)=\left(1-|z|^{2}\right) Q_{\bar{z}}+\left(1-|z|^{2}\right)^{2} P_{\bar{z}} .
$$

Thus

$$
\left(1-|z|^{2}\right)^{2} I \leq B^{t}(z, z) \leq\left(1-|z|^{2}\right) I
$$

in particular $B^{t}(z, z)$ is a positive operator. Actually $\otimes^{s} B^{t}(z, z)$ is positive on $\otimes^{s} V^{\prime}$. To prove this we need an elementary observation.

Lemma 2.5. Let $H_{1}$ and $H_{2}$ be Hilbert spaces. Let $A$ and $B$ be positive operators on $H_{1}$ and $H_{2}$ respectively. Then the operator $A \otimes B$ is positive on the induced Hilbert space $H_{1} \otimes H_{2}$.

Remark 2.6. Since $B^{t}(z, z)$ is positive on $V^{\prime}$ we have now that $\otimes^{s} B^{t}(z, z)$ is positive for $s=0,1,2, \ldots$.

\subsection{The norm of $z^{\alpha}$ in the Bergman space $L_{a}^{2}\left(d \iota_{v}\right)$}

Let $\alpha=\left(\alpha_{1}, \alpha_{2}, \ldots, \alpha_{d}\right)$ denote ordered $d$-tuples of non-negative integers $\alpha_{i}$ and denote $|\alpha|=\alpha_{1}+\cdots+\alpha_{d}$. Then the polynomials $\left\{z^{\alpha}\right\}$ forms an orthogonal basis for $L_{a}^{2}\left(d \iota_{v}\right)$ and

$$
\left\|z^{\alpha}\right\|_{\nu}^{2}=\int_{\mathrm{B}}\left|z_{1}^{\alpha_{1}} \cdot z_{2}^{\alpha_{2}} \cdots z_{d}^{\alpha_{d}}\right|^{2} d l_{v}(z)=\frac{\alpha_{1} ! \alpha_{2} ! \cdots \alpha_{d} !}{(v)_{|\alpha|}}
$$

where $(v)_{|\alpha|}=v(v+1) \cdots(v+|\alpha|-1)=\Gamma(v+|\alpha|) / \Gamma(v),(v)_{0}=1$, is the Pochammer symbol.

2.4. Some remarks on boundedness, compactness and $\mathscr{S}_{2}$

Consider the bilinear Hankel form $H_{F}^{s}$ with symbol $F$. First observe that the operator norm of the corresponding operator $\bar{A}_{F}^{s}$ equals

$$
\left\|H_{F}^{s}\right\|=\sup _{\|f\|_{v}=\|g\|_{v}=1}\left|H_{F}^{s}(f, g)\right| .
$$

If $\bar{A}_{F}^{s}$ is compact and $\left\{g_{n}\right\}_{n=1}^{\infty} \subset L_{a}^{2}\left(d \iota_{\nu}\right)$, with $\left\|g_{n}\right\|_{\nu}=1, g_{n} \rightarrow 0$ weakly as $n \rightarrow \infty$, then there is a sequence $\left\{c_{n}\right\}_{n=1}^{\infty}$ of positive numbers such that

$$
\left|H_{F}^{s}\left(f, g_{n}\right)\right| \leq c_{n}\|f\|_{\nu}
$$

for all $n$. Also $c_{n} \rightarrow 0$ as $n \rightarrow \infty$. On the other hand, if $\left\{A_{n}\right\}_{n=1}^{\infty}$ is a sequence of compact bilinear forms on $L_{a}^{2}\left(d \iota_{v}\right) \otimes L_{a}^{2}\left(d \iota_{v}\right)$ such that $A_{n} \rightarrow H_{F}^{s}$ in operator 
norm, then $H_{F}^{s}$ is compact. Also $H_{F}^{s}$ is of Hilbert-Schmidt class $\mathscr{S}_{2}$ if and only if

$$
\left\|H_{F}^{s}\right\|_{\mathscr{S}_{2}}^{2}=\sum_{|\alpha|=0}^{\infty} \sum_{|\beta|=0}^{\infty}\left|H_{F}^{s}\left(e_{\alpha}, e_{\beta}\right)\right|^{2}<\infty
$$

where $e_{\alpha}=z^{\alpha} /\left\|z^{\alpha}\right\|_{\nu}$. In addition, if $A$ is a bilinear form on $L_{a}^{2}\left(d \iota_{v}\right) \otimes L_{a}^{2}\left(d \iota_{v}\right)$ of Hilbert-Schmidt class, then $A$ is compact.

\section{The Banach space $\mathscr{H}_{v, s}^{p}$}

Let $L_{\nu, s}^{p}$, for $1<p<\infty$, be the space of measurable functions $S: \mathrm{B} \rightarrow \odot^{s} V^{\prime}$ such that

$$
\|S\|_{\nu, s, p}=\left(\int_{\mathrm{B}}\left\langle\left(1-|z|^{2}\right)^{2 v} \otimes^{s} B^{t}(z, z) S(z),\left.S(z)\right|^{p / 2} d \iota(z)\right)^{1 / p}<\infty .\right.
$$

Then $L_{v, s}^{p}$ is a Banach space. The closed subspace of holomorphic functions in $L_{v, s}^{p}$ is denoted by $\mathscr{H}_{v, s}^{p}$.

\subsection{Transformation properties of $H_{F}^{s}$}

Define an action $\pi_{v}$ of $G$ on $L_{a}^{2}\left(d \iota_{v}\right)$ by

$$
\pi_{v}: g \in G, f(w) \rightarrow f\left(g^{-1} w\right)\left(J_{g^{-1}}(w)\right)^{v /(d+1)} .
$$

Remark 3.1. Let $z \in \mathrm{B}$. Then $\Re(1-\langle w, z\rangle) \geq(1-|z|)>0$ for all $w \in \mathbf{B}$ so that $(1-\langle w, z\rangle)^{\alpha}$ can be defined as a holomorphic function in $w$ for any real $\alpha$. Thus for any $g \in G$, writing $g=U \varphi_{z}$ where $U \in \mathscr{U}(d)$ and $\varphi_{z}$ is the linear fractional mapping (8), we let, according to Proposition 2.2,

$$
\left(J_{g^{-1}}(w)\right)^{v /(d+1)}=\left((-1)^{d}\left(1-|z|^{2}\right)^{(d+1) / 2} \operatorname{det} U\right)^{v /(d+1)} \cdot(1-\langle w, z\rangle)^{-v}
$$

which then defines a holomorphic function in $w$.

Actually $\pi_{v}: g \rightarrow \pi_{v}(g)$ is a projective unitary representation on $L_{a}^{2}\left(d \iota_{v}\right)$, that is $\left\|\pi_{\nu}(g) f\right\|_{\nu}=\|f\|_{\nu}$ and $\pi_{v}\left(g_{1} g_{2}\right)=C\left(g_{1}, g_{2}\right) \pi_{v}\left(g_{1}\right) \pi_{v}\left(g_{2}\right)$ for some constant $C\left(g_{1}, g_{2}\right)$. This yields the following equality of two operator norms

$$
\left\|H_{F}^{s}\left(\pi_{\nu}(g)(\cdot), \pi_{\nu}(g)(\cdot)\right)\right\|=\left\|H_{F}^{s}\right\| .
$$

Define an action $\pi_{v, s}$ on $\mathscr{H}_{v, s}^{2}$ by

$$
\pi_{v, s}: g \in G, S(z) \rightarrow\left(\otimes^{s}\left(d g^{-1}(z)\right)^{t}\right) S\left(g^{-1} z\right)\left(J_{g^{-1}}(z)\right)^{2 v /(d+1)} .
$$


Then

$$
H_{F}^{s}\left(\pi_{v}(g) f_{1}, \pi_{v}(g) f_{2}\right)=H_{S}^{s}\left(f_{1}, f_{2}\right)
$$

where $S(z)=\pi_{v, s}\left(g^{-1}\right) F(z)$. Equation (16) is a consequence of Lemma 3.2 below. Define an action $\pi_{v}(\cdot) \otimes \pi_{v}(\cdot)$ on $L_{a}^{2}\left(d \iota_{v}\right) \otimes L_{a}^{2}\left(d \iota_{v}\right)$ by

$$
\begin{aligned}
\pi_{v} \otimes \pi_{v} & : g \in G,\left(f_{1}\left(w_{1}\right), f_{2}\left(w_{2}\right)\right) \\
& \rightarrow f_{1}\left(g^{-1} w_{1}\right) f_{2}\left(g^{-1} w_{2}\right)\left(J_{g^{-1}}\left(w_{1}\right)\right)^{v /(d+1)}\left(J_{g^{-1}}\left(w_{2}\right)\right)^{v /(d+1)}
\end{aligned}
$$

The following invariance property of the Transvectant is proved in [11], see also [15].

LEMMA 3.2. Let $\pi_{v, s}$ and $\pi_{v}(\cdot) \otimes \pi_{v}(\cdot)$ be the representations given by (15) and (17) respectively. Let $g \in G$. Then

$$
\mathscr{T}_{s}\left(\pi_{v}(g) \otimes \pi_{v}(g)\right)\left(f_{1}, f_{2}\right)=\pi_{v, s}(g) \mathscr{T}_{s}\left(f_{1}, f_{2}\right) .
$$

REMARK 3.3. It follows from Theorem 4.1 that $\mathscr{T}_{s}$ takes values in $\mathscr{H}_{v, s}^{2}$. In fact, Theorem 4.1 shows that $\mathscr{T}_{s}: L_{a}^{2}\left(d \iota_{v}\right) \otimes L_{a}^{2}\left(d \iota_{v}\right) \rightarrow \mathscr{H}_{v, s}^{2}$ is a bounded bilinear form.

REMARK 3.4. As a consequence of Lemma 3.2 we have (16), namely

$$
\begin{aligned}
H_{F}^{s}\left(\left(\pi_{v}(g) \otimes \pi_{v}(g)\right)\left(f_{1}, f_{2}\right)\right) & =\left\langle\mathscr{T}_{s}\left(\pi_{v}(g) \otimes \pi_{v}(g)\right)\left(f_{1}, f_{2}\right), F\right\rangle_{\nu, s, 2} \\
& =\left\langle\pi_{v, s}(g) \mathscr{T}_{s}\left(f_{1}, f_{2}\right), F\right\rangle_{\nu, s, 2} \\
& =\left\langle\mathscr{T}_{s}\left(f_{1}, f_{2}\right), \pi_{v, s}\left(g^{-1}\right) F\right\rangle_{\nu, s, 2}
\end{aligned}
$$

which gives the result if we observe that $S=\pi_{v, s}\left(g^{-1}\right) F$.

\subsection{Reproducing kernel of the space $\mathscr{H}_{v, s}^{2}$}

LEMMA 3.5. The reproducing kernel of $\mathscr{H}_{v, s}^{2}$ is, up to a nonzero constant,

$$
K_{v, s}(z, w)=(1-\langle z, w\rangle)^{-2 v} \otimes^{s}\left(B^{t}(z, w)\right)^{-1} .
$$

Namely, for any $f \in \mathscr{H}_{v, s}^{2}$ and any $v \in \odot^{s} V^{\prime}$ it holds that

$$
\begin{aligned}
\langle f(z), v\rangle & =c\left\langle f(\cdot), K_{v, s}(\cdot, z) v\right\rangle_{v, s, 2} \\
& =c \int_{\mathrm{B}}\left\langle\left(1-|w|^{2}\right)^{2 v} \otimes^{s} B^{t}(w, w) f(w), K_{v, s}(w, z) v\right\rangle d \iota(w) .
\end{aligned}
$$


Proof. For any $v \in \odot^{s} V^{\prime}$ we prove that $f \rightarrow\langle f(z), v\rangle$ is a bounded functional on $\mathscr{H}_{v, s}^{2}$. It follows then by Riesz lemma that there exists a function $R(z, w): \odot^{s} V^{\prime} \rightarrow \odot^{s} V^{\prime}$ such that $\langle f(z), v\rangle=\langle f, R(\cdot, z) v\rangle_{v, s, 2}$. Let $f \in$ $\mathscr{H}_{v, s}^{2}$ and let $z \in \mathrm{B}$. Since $z \rightarrow\|f(z)\|$ is subharmonic then

$$
\|f(z)\| \leq C_{d, r, v} \int_{z+r \mathrm{~B}}\|f(w)\| d \iota_{2 v}(w)
$$

so by Jensen's inequality

$$
\|f(z)\|^{2} \leq C_{d, r, v}^{\prime} \int_{z+r \mathrm{~B}}\|f(w)\|^{2} d \iota_{2 v}(w)
$$

if $\overline{z+r \mathrm{~B}} \subset \mathrm{B}$. On the other hand, there is a constant $d_{r}>0$ such that $d_{r} I \leq$ $\otimes^{s} B^{t}(w, w)$ for all $w \in \overline{z+r \mathrm{~B}}$. Hence

$$
\|f(z)\|^{2} \leq D_{d, r, v} \int_{z+r \mathrm{~B}}\left\langle\left(1-|z|^{2}\right)^{2 v} \otimes^{s} B^{t}(w, w) f(w), f(w)\right\rangle d_{\iota}(w)
$$

so that $f \rightarrow\langle f(z), v\rangle$ is bounded. Then the reproducing property at $z=0$ reads as

$$
\langle f(0), v\rangle=\langle f(\cdot), R(\cdot, 0) v\rangle_{v, s, 2} .
$$

On the other hand, the space of $\odot^{s} V^{\prime}$-valued polynomials is dense in $\mathscr{H}_{v, s}^{2}$ and $\langle p(\cdot), v\rangle_{v, s, 2}=0$ for all homogeneous polynomials of degree $\geq 1$. Thus if

$$
f(z)=\sum_{m=0}^{\infty} f_{m}(z)
$$

where $f_{m}$ are homogeneous polynomials of degree $m$, then

$$
\langle f(\cdot), v\rangle_{v, s, 2}=\left\langle f_{0}(\cdot), v\right\rangle_{v, s, 2}=\langle f(0), v\rangle_{v, s, 2}=c^{\prime}\langle f(0), v\rangle .
$$

Therefore

$$
\langle f(\cdot), R(\cdot, 0) v\rangle_{v, s, 2}=\langle f(0), v\rangle=\frac{1}{c^{\prime}}\langle f(\cdot), v\rangle_{v, s, 2}
$$

so that $R(\cdot, 0)=c I$ with $c \neq 0$. Next we prove that $R(z, w)$ transforms under $G$ as follows

(18) $R(g z, g w)$

$$
=\left(\otimes^{s} d g(z)^{t}\right)^{-1} R(z, w)\left(\otimes^{s}\left(d g(w)^{t}\right)^{*}\right)^{-1}\left(J_{g}(z)\right)^{-2 v /(d+1)}\left(\overline{J_{g}(w)}\right)^{-2 v /(d+1)}
$$


where $g \in G$. Indeed, for all $F \in \mathscr{H}_{v, s}^{2}$

$$
\langle F(z), v\rangle=\int_{\mathrm{B}}\left\langle\left(1-|w|^{2}\right)^{2 v} \otimes^{s} B^{t}(w, w) F(w), R(w, z) v\right\rangle d \iota(w)
$$

from which it follows that for all $f \in L_{a}^{2}\left(d \iota_{v}\right)$

$$
\begin{gathered}
\left\langle J_{g}(z)^{2 v /(d+1)} \otimes^{s} d g(z)^{t} f(g z), v\right\rangle \\
=\int_{\mathrm{B}}\left\langle\left(1-|w|^{2}\right)^{2 v} \otimes^{s} B^{t}(w, w) J_{g}(w)^{2 v /(d+1)}\right. \\
\left.\otimes^{s} d g(w)^{t} f(g w), R(w, z) v\right\rangle d \iota(w) .
\end{gathered}
$$

On the other hand, it follows from (9)

$$
\begin{aligned}
& \left\langle f(g z),\left(\overline{J_{g}(z)}\right)^{2 v /(d+1)} \otimes^{s}\left(d g(z)^{t}\right)^{*} v\right\rangle \\
& =\int_{\mathrm{B}}\left\langle\otimes^{s} B^{t}(w, w) f(w), R(w, g z)\left(\overline{J_{g}(z)}\right)^{2 v /(d+1)} \otimes^{s}\left(d g(z)^{t}\right)^{*} v\right\rangle \frac{d \iota_{2 v}(w)}{c_{2 v}} \\
& =\int_{\mathrm{B}}\left\langle\otimes^{s} B^{t}(g w, g w) f(g w), R(g w, g z)\left(\overline{J_{g}(z)}\right)^{2 v /(d+1)} \otimes^{s}\left(d g(z)^{t}\right)^{*} v\right\rangle \\
& \cdot\left|\left(J_{g}(w)\right)^{2 v /(d+1)}\right|^{2} \frac{d \iota_{2 v}(w)}{c_{2 v}} \\
& =\int_{\mathrm{B}}\left\langle\left(1-|w|^{2}\right)^{2 v} \otimes^{s} B^{t}(w, w)\left(J_{g}(w)\right)^{2 v /(d+1)} \otimes^{s} d g(w)^{t} f(g w),\right. \\
& \left.\otimes^{s} d g(w)^{t} R(g w, g z) \otimes^{s}\left(d g(z)^{t}\right)^{*} v\right\rangle \\
& \cdot\left(J_{g}(z)\right)^{2 v /(d+1)}\left(\overline{J_{g}(w)}\right)^{2 v /(d+1)} d \iota(w) .
\end{aligned}
$$

Comparing this with (19) we get (18). Now both $R(z, w) / c$ and $K_{v, s}(z, w)$ satisfy the same transformation rule (18) and are identity operator at $z=0$. Thus they are the same for all $z, w \in \mathrm{B}$. This completes the proof of the lemma.

\section{The Besov space $\mathscr{B}_{v, s}$}

Let $s=1,2,3, \ldots$ and define

$$
\begin{aligned}
& \mathscr{B}_{v, s} \\
& =\left\{f: \mathrm{B} \rightarrow \mathrm{C} \text { holomorphic, } \int_{\mathrm{B}}\left\langle\otimes^{s} B^{t}(z, z) \partial^{s} f(z), \partial^{s} f(z)\right\rangle d \iota_{v}(z)<+\infty\right\} .
\end{aligned}
$$


The space $\mathscr{B}_{v, s}$ is called a Besov space. It is a Hilbert space, equipped with the inner product $\langle\cdot, \cdot\rangle_{v, s}$ given by

$$
\begin{aligned}
\langle f, g\rangle_{v, s}=f(0) \overline{g(0)}+\cdots+ & \left\langle\left(\partial^{(s-1)} f\right)(0),\left(\partial^{(s-1)} g\right)(0)\right\rangle \\
& +\int_{B}\left\langle\otimes^{s} B^{t}(z, z) \partial^{s} f(z), \partial^{s} g(z)\right\rangle d \iota_{v}(z) .
\end{aligned}
$$

Actually $\mathscr{B}_{v, s}=L_{a}^{2}\left(d \iota_{v}\right)$, namely they are equal as sets and their norms are equivalent, as is shown below.

Theorem 4.1. There exist constants $C_{v, s}, D_{v, s}>0$ such that

$$
C_{\nu, s} \cdot\|f\|_{v} \leq\|f\|_{\nu, s} \leq D_{\nu, s} \cdot\|f\|_{\nu}
$$

for all holomorphic $f: \mathrm{B} \rightarrow \mathrm{C}$.

We need first some elementary lemmas.

LeMMA 4.2. Let $f_{m}$ and $f_{n}$ be homogeneous holomorphic polynomials of degree $m$ and $n$ respectively, with $m \neq n$. Then $\left\langle f_{m}, f_{n}\right\rangle_{v, s}=0$.

Proof. Let $0<\theta<2 \pi$. Then $e^{i \theta} \neq 1$. Since $f_{m}$ is a homogeneous polynomial of degree $m$ we have $f_{m}\left(e^{i \theta} z\right)=e^{i m \theta} f_{m}(z)$. Given $m$ and $n$ with $m \neq n$, it is enough to prove that

$$
\left\langle f_{m}, f_{n}\right\rangle_{\nu, s}=e^{i(m-n) \theta}\left\langle f_{m}, f_{n}\right\rangle_{\nu, s}
$$

The case $s=0$ follows directly from the homogeneity. Now consider the case $s=1$. It is easy to see that $B^{t}(z, z)=B^{t}\left(e^{-i \theta} z, e^{-i \theta} z\right)$. By the chain rule and homogeneity it follows that

$$
\left(\partial f_{m}\right)\left(e^{i \theta} w\right)=e^{-i \theta} \partial\left(f_{m}\left(e^{i \theta} \cdot\right)\right)(w)=e^{i(m-1) \theta}\left(\partial f_{m}\right)(w)
$$

so that the equation (20) holds for $s=1$. The cases $s=2,3, \ldots$ now follow in the same way if we first notice that $\left(\partial^{s} f_{m}\right)\left(e^{i \theta} w\right)=e^{i(m-s) \theta}\left(\partial^{s} f_{m}\right)(w)$. This completes the proof.

We recall now a result from Rudin (Theorem 12.2.8 in [20]). Consider the space $\mathscr{P}_{m}$ of all homogeneous holomorphic polynomials of degree $m$ on B with the natural group action of the unitary group $\mathscr{U}(d)$ :

$$
\left(\pi_{g} f\right)(z)=f\left(g^{-1} z\right), \quad f \in \mathscr{P}_{m}, \quad g \in \mathscr{U}(d) .
$$

Then $\left(\mathscr{P}_{m}, \pi_{g}\right)$ is a unitary irreducible representation of $\mathscr{U}(d)$. As a consequence of Schur's lemma (Theorem 1.10 in [2]) we have 
Lemma 4.3. Let $m$ be a non-negative integer. Then there exists a positive constant $C_{v, s, m}$ such that

$$
\left\|f_{m}\right\|_{v, s}=C_{v, s, m} \cdot\left\|f_{m}\right\|_{v}
$$

for all $f_{m} \in \mathscr{P}_{m}$.

Remark 4.4. Actually, this lemma is a special case of the result in exercise 1.16.7 in [2].

Now we can prove the norm-equivalence of $\mathscr{B}_{v, s}$ and $L_{a}^{2}\left(d \iota_{v}\right)$.

Proof of Theorem 4.1. It is enough to prove the theorem for $f$ with $f(0)=\cdots=\partial^{s-1} f(0)=0$. Write $f=\sum_{m=0}^{\infty} f_{m}$ where $f_{m} \in \mathscr{P}_{m}$. By Lemma 4.2 we have that $\left\{f_{m}\right\}_{m=0}^{\infty}$ is an orthogonal set in both $L_{a}^{2}\left(d \iota_{v}\right)$ and $\mathscr{B}_{v, s}$. Also, by Lemma 4.3 we have $\left\|f_{m}\right\|_{v, s}=C_{v, s, m} \cdot\left\|f_{m}\right\|_{\nu}$ where $C_{v, s, m}$ does not depend on $f_{m}$ of degree $m$. We compute $C_{v, s, m}$ and prove that there exist positive constants $C_{v, s}$ and $D_{v, s}$ such that

$$
C_{\nu, s} \leq C_{v, s, m} \leq D_{v, s}
$$

for all $m$. We may assume that $m \geq s$. Take $f_{m}(z)=z_{1}^{m}$. We shall calculate

$$
\left\|f_{m}\right\|_{v, s}^{2}=\int_{\mathbf{B}}\left\langle\otimes^{s} B^{t}(z, z) \partial^{s} f_{m}(z), \partial^{s} f_{m}(z)\right\rangle d \iota_{v}(z) .
$$

First observe that

$$
\begin{aligned}
\left\langle\otimes^{s} B^{t}(z, z) \partial^{s} f_{m}(z), \partial^{s} f_{m}(z)\right\rangle & \\
& =\left\langle\otimes^{s} B^{t}(z, z)\left(\partial_{1}^{s} z_{1}^{m}\right) \otimes^{s} d z_{1},\left(\partial_{1}^{s} z_{1}^{m}\right) \otimes^{s} d z_{1}\right\rangle \\
& =\left\langle B^{t}(z, z)\left(\partial_{1}^{s} z_{1}^{m}\right) d z_{1},\left(\partial_{1}^{s} z_{1}^{m}\right) d z_{1}\right\rangle \cdot\left\langle B^{t}(z, z) d z_{1}, d z_{1}\right\rangle^{s-1} \\
& =\frac{\Gamma(m+1)^{2}}{\Gamma(m-s+1)^{2}}\left(1-|z|^{2}\right)^{s}\left(1-\left|z_{1}\right|^{2}\right)^{s}\left|z_{1}\right|^{2(m-s)} .
\end{aligned}
$$

We have

$$
\begin{aligned}
& C_{v} \int_{\mathrm{B}}\left|z_{1}\right|^{2(m-s)}\left(1-\left|z_{1}\right|^{2}\right)\left(1-|z|^{2}\right)^{v+s} d \iota(z)= \\
& \int_{\left|z_{1}\right|<1}\left|z_{1}\right|^{2(m-s)}\left(1-\left|z_{1}\right|^{2}\right)^{s} \int_{\left|z^{\prime}\right|<\sqrt{1-\left|z_{1}\right|^{2}}}\left(1-\left|z_{1}\right|^{2}-\left|z^{\prime}\right|^{2}\right)^{v+s-d-1} d m\left(z^{\prime}\right) d m\left(z_{1}\right)
\end{aligned}
$$

and

$$
\int_{\left|z^{\prime}\right|<\sqrt{1-\left|z_{1}\right|^{2}}}\left(1-\left|z_{1}\right|^{2}-\left|z^{\prime}\right|^{2}\right)^{v+s-d-1} d m\left(z^{\prime}\right)=C_{v}^{\prime} \cdot\left(1-\left|z_{1}\right|^{2}\right)^{v+s-2} .
$$


Since

$$
\begin{array}{r}
\int_{\left|z_{1}\right|<1}\left|z_{1}\right|^{2(m-s)}\left(1-\left|z_{1}\right|^{2}\right)^{s}\left(1-\left|z_{1}\right|^{2}\right)^{s+v-2} d m\left(z_{1}\right) \\
=C_{v}^{\prime \prime} \cdot \frac{\Gamma(m-s+1) \Gamma(v+2 s-1)}{\Gamma(m+s+v)}
\end{array}
$$

we get

$$
\left\|f_{m}\right\|_{v, s}^{2}=a_{v} \cdot \frac{\Gamma(m+1)^{2} \Gamma(v+2 s-1)}{\Gamma(m-s+1) \Gamma(m+s+v)} .
$$

On the other hand

$$
\left\|f_{m}\right\|_{v}^{2}=\frac{\Gamma(m+1) \Gamma(v)}{\Gamma(m+v)}
$$

so that

$$
C_{v, s, m}^{2}=\frac{\left\|f_{m}\right\|_{v, s}^{2}}{\left\|f_{m}\right\|_{v}^{2}}=a_{v} \cdot \frac{\Gamma(m+1) \Gamma(v+2 s-1) \Gamma(m+v)}{\Gamma(m-s+1) \Gamma(m+s+v) \Gamma(v)} .
$$

For $m \geq s$ we have

$$
\begin{aligned}
\frac{\Gamma(m+1) \Gamma(m+v)}{\Gamma(m-s+1) \Gamma(m+s+v)} & =\frac{m(m-1) \cdots(m-s+1)}{(m+s+v-1) \cdots(m+v)} \\
& =\frac{\left(1-\frac{1}{m}\right) \cdots\left(1-\frac{s-1}{m}\right)}{\left(1+\frac{s+v-1}{m}\right) \cdots\left(1+\frac{v}{m}\right)}
\end{aligned}
$$

so that

$$
b_{v, s}=\frac{\left(1-\frac{1}{s}\right) \cdots\left(1-\frac{s-1}{s}\right)}{\left(1+\frac{s+v-1}{s}\right) \cdots\left(1+\frac{v}{s}\right)} \leq \frac{\Gamma(m+1) \Gamma(m+v)}{\Gamma(m-s+1) \Gamma(m+s+v)} \leq 1 .
$$

So (21) follows by putting

$$
C_{v, s}=\sqrt{\frac{a_{v} \cdot b_{v, s} \cdot \Gamma(v+2 s-1)}{\Gamma(v)}}
$$

and

$$
D_{v, s}=\sqrt{\frac{a_{v} \cdot \Gamma(v+2 s-1)}{\Gamma(v)}} .
$$




\section{Boundedness}

\subsection{The Banach space $\mathscr{H}_{\nu, s}^{\infty}$}

Denote by $L_{v, s}^{\infty}$ the space of functions $F: \mathrm{B} \rightarrow \odot^{s} V^{\prime}$ such that

$$
\|F\|_{v, s, \infty}=\sup _{z \in B}\left\langle\left(1-|z|^{2}\right)^{2 v} \otimes^{s} B^{t}(z, z) F(z),\left.F(z)\right|^{1 / 2}<\infty .\right.
$$

If we write $\|F\|_{v, s, \infty}=\sup _{z \in B}\|S(z)\|_{W}$ where

$$
\|S(z)\|_{W}=\left\|\left(\left(1-|z|^{2}\right)^{2 v} \otimes^{s} B^{t}(z, z)\right)^{1 / 2} F(z)\right\|
$$

and $W=\odot^{s} V^{\prime}$, then $L_{v, s}^{\infty}$ is a Banach space since it is easy to see that, if $S_{n}: \mathrm{B} \rightarrow W$ satisfies

$$
\sum_{n=1}^{\infty} \sup _{z \in B}\left\|S_{n}(z)\right\|_{W}<\infty
$$

then there is a $S: \mathrm{B} \rightarrow W$ with $\sup _{z \in \mathrm{B}}\|S(z)\|_{W}<\infty$ such that

$$
\sup _{z \in \mathrm{B}}\left\|S(z)-\sum_{n=1}^{N} S_{n}(z)\right\|_{W} \rightarrow 0 \quad \text { as } \quad N \rightarrow \infty .
$$

The closed subspace of holomorphic functions in $L_{\nu, s}^{\infty}$ is denoted by $\mathscr{H}_{\nu, s}^{\infty}$.

\subsection{Proof of Theorem 1.1(a)}

Proof OF SUfFiciency. The Hankel form in (7) can be written as a sum of certain integrals, we estimate each one, as follows,

$$
\begin{aligned}
& \left|\int_{\mathrm{B}}\left\langle\left(1-|z|^{2}\right)^{2 v} \otimes^{s} B^{t}(z, z) \partial^{k} f(z) \otimes \partial^{s-k} g(z), F(z)\right\rangle d \iota(z)\right| \leq \\
& \|F\|_{\nu, s, \infty} \cdot \int_{\mathrm{B}}\left\langle\otimes^{s} B^{t}(z, z) \partial^{k} f(z) \otimes \partial^{s-k} g(z),\left.\partial^{k} f(z) \otimes \partial^{s-k} g(z)\right|^{1 / 2} \frac{d \iota_{\nu}(z)}{c_{\nu}}\right.
\end{aligned}
$$

and

$$
\begin{aligned}
& \left\langle\otimes^{s} B^{t}(z, z) \partial^{k} f(z) \otimes \partial^{s-k} g(z), \partial^{k} f(z) \otimes \partial^{s-k} g(z)\right\rangle \\
& =\left\langle\otimes^{k} B^{t}(z, z) \partial^{k} f(z), \partial^{k} f(z)\right\rangle \cdot\left\langle\otimes^{s-k} B^{t}(z, z) \partial^{s-k} g(z), \partial^{s-k} g(z)\right\rangle
\end{aligned}
$$

so that

$$
\begin{aligned}
& \left|\int_{\mathbf{B}}\left\langle\left(1-|z|^{2}\right)^{2 v} \otimes^{s} B^{t}(z, z) \partial^{k} f(z) \otimes \partial^{s-k} g(z), F(z)\right\rangle d \iota(z)\right| \\
& \leq c_{v}^{\prime} \cdot\|F\|_{\nu, s, \infty} \cdot\|f\|_{v, k} \cdot\|g\|_{\nu, s-k} \leq C_{v, s} \cdot\|F\|_{s, \infty} \cdot\|f\|_{\nu} \cdot\|g\|_{\nu},
\end{aligned}
$$


where the last inequality follows from Theorem 4.1.

For notational convenience we denote

$$
\langle u, v\rangle_{z}=\left\langle\otimes^{s} B^{t}(z, z) u, v\right\rangle
$$

where $u, v \in \odot^{s} V^{\prime}$, and it defines an inner product on $\odot^{s} V^{\prime}$.

Proof OF NECESSITY. Let $v \in \odot^{s} V^{\prime}$. By Lemma 3.5 we have

$$
\langle F(0), v\rangle=c \int_{\mathrm{B}}\left\langle\left(1-|w|^{2}\right)^{2 v} \otimes^{s} B^{t}(w, w) F(w), v\right\rangle d \iota(w) .
$$

We may write

$$
v=\sum_{|i|=s} v_{i} e_{1}^{i_{1}} \odot \cdots \odot e_{d}^{i_{d}}
$$

where $i=\left(i_{1}, \ldots, i_{d}\right)$ and $v_{i} \in \mathrm{C}$. Take

$$
f(w)=\sum_{|i|=s} w_{1}^{i_{1}} \cdots w_{d}^{i_{s}} \cdot v_{i} \quad \text { and } \quad g(w)=1 .
$$

Then $f, g \in L_{a}^{2}\left(d \iota_{v}\right)$. By (6)

$$
\begin{aligned}
\mathscr{T}_{s}(f, g)(w) & =\sum_{k=0}^{s}\left(\begin{array}{l}
s \\
k
\end{array}\right)(-1)^{s-k} \frac{\partial^{k} f(w) \odot \partial^{s-k} g(w)}{(\nu)_{k}(v)_{s-k}} \\
& =\left(\begin{array}{l}
s \\
0
\end{array}\right) \frac{\partial^{s} f(w) \odot g(w)}{(v)_{s}(v)_{0}}
\end{aligned}
$$

where

$$
\partial^{s} f(w)=\sum_{|i|=s} \partial^{s}\left(w_{1}^{i_{1}} \cdots w_{d}^{i_{d}}\right) \cdot v_{i}=\sum_{|i|=s} s ! \cdot v_{i} e_{1}^{i_{1}} \odot \cdots \odot e_{d}^{i_{d}}=s ! v
$$

so that

$$
\mathscr{T}_{s}(f, g)(w)=\frac{s !}{(v)_{s}} v .
$$

Hence

$$
|\langle F(0), v\rangle|^{2}=c^{2}(v)_{s}^{2} \cdot \frac{1}{(s !)^{2}}\left|H_{F}^{s}(f, g)\right|^{2}
$$

so that

$$
|\langle F(0), v\rangle|^{2} \leq C_{v, s}\left\|H_{F}^{s}\right\|^{2}\|f\|_{\nu}^{2}\|g\|_{\nu}^{2} \leq C_{\nu, s}\left\|H_{F}^{s}\right\|^{2}\|v\|^{2} .
$$


Define

$$
S(w)=\left(\pi_{v, s}\left(\varphi_{z}\right) F\right)(w)=\left(\otimes^{s} \varphi_{z}^{\prime}(w)^{t}\right) F\left(\varphi_{z}(w)\right)\left(J_{\varphi_{z}}(w)\right)^{2 v /(d+1)} .
$$

Then $S: \mathrm{B} \rightarrow \odot^{s} V^{\prime}$ is holomorphic. Also by equations (14) and (16)

$$
\left\|H_{S}^{s}\right\|=\left\|H_{F}^{s}\right\|<\infty
$$

so by (23) with $F$ replaced by $S$

$$
|\langle S(0), v\rangle|^{2} \leq C\left\|H_{S}^{s}\right\|^{2}\|v\|^{2}=C\left\|H_{F}^{s}\right\|^{2}\|v\|^{2} .
$$

Now

$$
S(0)=\left(\otimes^{s} \varphi_{z}^{\prime}(0)^{t}\right) F(z)\left(J_{\varphi_{z}}(0)\right)^{2 v /(d+1)} .
$$

Since $-\varphi_{z}^{\prime}(0)^{t}=s_{z}^{2} P_{\bar{z}}+s_{z} Q_{\bar{z}} \geq 0$ then $\left(-\varphi_{z}^{\prime}(0)^{t}\right)^{2}=B^{t}(z, z)$ and by the uniqueness of positive square root $B^{t}(z, z)^{1 / 2}=-\varphi_{z}^{\prime}(0)^{t}$. Thus

$$
\left(\otimes^{s} B^{t}(z, z)\right)^{1 / 2}=\otimes^{s} B^{t}(z, z)^{1 / 2}=(-1)^{s} \otimes^{s} \varphi_{z}^{\prime}(0)^{t} .
$$

Hence

$$
S(0)=\rho\left(1-|z|^{2}\right)^{v}\left(\otimes^{s} B^{t}(z, z)\right)^{1 / 2} F(z),
$$

where $|\rho|=1$, so that (24) becomes

$$
\left|\left\langle F(z),\left(\otimes^{s} B^{t}(z, z)\right)^{1 / 2} v\right\rangle\right|^{2} \leq C\left\|H_{F}^{s}\right\|^{2}\left\|\left(\otimes^{s} B^{t}(z, z)\right)^{-1 / 2} v\right\|_{z}^{2}\left(1-|z|^{2}\right)^{-2 v} \text {. }
$$

Observe that

$$
\left\langle F(z),\left(\otimes^{s} B^{t}(z, z)\right)^{1 / 2} v\right\rangle=\left\langle F(z),\left(\otimes^{s} B^{t}(z, z)\right)^{-1 / 2} v\right\rangle_{z}
$$

so the result follows from Riesz lemma, for the inner product $\langle\cdot, \cdot\rangle_{z}$.

\section{Compactness and Hilbert-Schmidt properties}

\subsection{Compactness}

In this subsection we prove Theorem 1.1 (b).

REMARK 6.1. Let $\left\{e_{1}, \ldots, e_{d}\right\}$ be a basis for $V^{\prime}$. Then we can write

$$
F(z)=\sum_{i_{1}+\cdots+i_{d}=s} F_{i}(z) e_{1}^{i_{1}} \odot \cdots \odot e_{d}^{i_{d}}
$$

where $i=\left(i_{1}, \ldots, i_{d}\right)$ and $F_{i}: \mathrm{B} \rightarrow \mathrm{C}$ are holomorphic. Also

$$
F_{i}(z)=\sum_{m=0}^{\infty} p_{m}^{(i)}(z)
$$


where $p_{m}^{(i)}$ are homogeneous holomorphic polynomials of degree $m$.

To prove the sufficiency of Theorem 1.1(b) we need the following result.

Lemma 6.2. Let $F: \mathrm{B} \rightarrow \odot^{s} V^{\prime}$ be holomorphic with the property

$$
\left\langle\left(1-|z|^{2}\right)^{2 v} \otimes^{s} B^{t}(z, z) F(z), F(z)\right\rangle \rightarrow 0 \quad \text { if }|z| \nearrow 1 .
$$

Let $\varepsilon>0$ be given. Then there exists a number $r^{\prime}$ with $0<r^{\prime}<1$ and a natural number $N$ such that

$$
\left\|F-P_{N}\right\|_{v, s, \infty}<\varepsilon
$$

where

$$
P_{N}(z)=\sum_{|i|=s} \sum_{m=0}^{N} p_{m}^{(i)}\left(r^{\prime} z\right) e_{1}^{i_{1}} \odot \cdots \odot e_{d}^{i_{d}} .
$$

REMARK 6.3. Remember that we have already defined

$$
\|F\|_{v, s, \infty}=\sup _{z \in \mathrm{B}}\left\langle\left(1-|z|^{2}\right)^{2 v} \otimes^{s} B^{t}(z, z) F(z),\left.F(z)\right|^{1 / 2}\right.
$$

for holomorphic $F: \mathrm{B} \rightarrow \odot^{s} V^{\prime}$.

Remark 6.4. Let $H_{1}$ and $H_{2}$ be Hilbert spaces and let $A_{1}, B_{1}: H_{1} \rightarrow H_{1}$ and $A_{2}, B_{2}: H_{2} \rightarrow H_{2}$ be positive operators. Then

$$
\begin{aligned}
\left(A_{1}-B_{1}\right) \otimes\left(A_{2}+B_{2}\right)+\left(A_{1}+B_{1}\right) \otimes & \left(A_{2}-B_{2}\right) \\
& =2\left(A_{1} \otimes A_{2}-B_{1} \otimes B_{2}\right) .
\end{aligned}
$$

Thus it follows from (25) that

$$
A_{1} \geq B_{1}, A_{2} \geq B_{2} \Longrightarrow A_{1} \otimes A_{2} \geq B_{1} \otimes B_{2} .
$$

Proof of Lemma 6.2. Let $\varepsilon>0$ be given. Then there exists $0<r_{0}<1$ such that

$$
\sup _{r_{0}<|z|<1}\left\langle\left(1-|z|^{2}\right)^{2 v} \otimes^{s} B^{t}(z, z) F(z), F(z)\right\rangle<\frac{\varepsilon^{2}}{32} .
$$

Define $F_{r}(z)=F(r z)$ where $0<r<1$. Since $P_{r \bar{z}}=P_{\bar{z}}$ then

$$
B^{t}(r z, r z)=\left(1-r^{2}|z|^{2}\right)\left(I-r^{2}|z|^{2} P_{r \bar{z}}\right) \geq B^{t}(z, z)
$$


for all $0<r<1$. By (26) it then follows that

$$
\otimes^{s} B^{t}(r z, r z) \geq \otimes^{s} B^{t}(z, z)
$$

for all $0<r<1$. Hence,

$$
\begin{aligned}
\left\langle\left(1-|z|^{2}\right)^{2 v} \otimes^{s} B^{t}(z, z)\right. & \left.F_{r}(z), F_{r}(z)\right\rangle \\
& \leq\left\langle\left(1-|r z|^{2}\right)^{2 v} \otimes^{s} B^{t}(r z, r z) F(r z), F(r z)\right\rangle .
\end{aligned}
$$

Then it follows from the inequalities

$$
\begin{aligned}
&\left\langle\otimes^{s} B^{t}(z, z)\right.\left.\left(F(z)-F_{r}(z)\right), F(z)-F_{r}(z)\right\rangle \\
& \leq\left\langle\otimes^{s} B^{t}(z, z) F(z), F(z)\right\rangle+\left\langle\otimes^{s} B^{t}(z, z) F_{r}(z), F_{r}(z)\right\rangle \\
&+2\left|\left\langle\otimes^{s} B^{t}(z, z) F(z), F_{r}(z)\right\rangle\right|
\end{aligned}
$$

and

$$
\begin{aligned}
& \left|\left\langle\otimes^{s} B^{t}(z, z) F(z), F_{r}(z)\right\rangle\right| \\
& \leq\left|\otimes^{s} B^{t}(z, z) F(z), F(z)\right|^{1 / 2}\left\langle\otimes^{s} B^{t}(z, z) F_{r}(z),\left.F_{r}(z)\right|^{1 / 2}\right.
\end{aligned}
$$

that, if $1>r>r_{1}=2 r_{0} /\left(1+r_{0}\right)$ and $R_{0}=\left(1+r_{0}\right) / 2$,

$$
\sup _{R_{0}<|z|<1}\left\langle\left(1-|z|^{2}\right)^{2 v} \otimes^{s} B^{t}(z, z)\left(F(z)-F_{r}(z)\right), F(z)-F_{r}(z)\right\rangle<\frac{\varepsilon^{2}}{8},
$$

since, if $r_{1}<r<1$,

$$
\begin{aligned}
\sup _{R_{0}<|z|<1} & \left\langle\left(1-|z|^{2}\right)^{2 v} \otimes^{s} B^{t}(z, z) F(r z), F(r z)\right\rangle \\
\quad \leq & \sup _{R_{0} r<|r z|<r}\left\langle\left(1-|r z|^{2}\right)^{2 v} \otimes^{s} B^{t}(r z, r z) F(r z), F(r z)\right\rangle \\
\quad \leq & \sup _{r_{0}<|r z|<1}\left\langle\left(1-|r z|^{2}\right)^{2 v} \otimes^{s} B^{t}(r z, r z) F(r z), F(r z)\right|<\frac{\varepsilon^{2}}{32} .
\end{aligned}
$$

As $F_{r} \rightarrow F$ uniformly, $r \rightarrow 1$, on every compact subset of $\mathrm{B}$, there is a number $r_{2}$ such that if $r_{2}<r<1$, then

$$
\sup _{|z| \leq R_{0}}\left\langle F(z)-F_{r}(z), F(z)-F_{r}(z)\right\rangle<\frac{\varepsilon^{2}}{8} .
$$


Since $B^{t}(z, z) \leq\left(1-|z|^{2}\right) I \leq I$ then (26) yields $\otimes^{s} B^{t}(z, z) \leq \otimes^{s} I$ so that if $r_{2}<r<1$, then

$$
\begin{aligned}
\sup _{|z| \leq R_{0}}\left\langle\left(1-|z|^{2}\right)^{2 v} \otimes^{s} B^{t}(z\right. & \left., z)\left(F(z)-F_{r}(z)\right), F(z)-F_{r}(z)\right\rangle \\
\leq & \sup _{|z| \leq R_{0}}\left\langle F(z)-F_{r}(z), F(z)-F_{r}(z)\right\rangle<\frac{\varepsilon^{2}}{8} .
\end{aligned}
$$

Hence for $\max \left(r_{1}, r_{2}\right)<r<1$ it holds that

$$
\begin{aligned}
& \left\|F-F_{r}\right\|_{v, s, \infty}^{2} \\
& \leq \sup _{|z| \leq R_{0}}\left\langle\left(1-|z|^{2}\right)^{2 v} \otimes^{s} B^{t}(z, z)\left(F(z)-F_{r}(z)\right), F(z)-F_{r}(z)\right\rangle \\
& \quad+\sup _{R_{0}<|z|<1}\left\langle\left(1-|z|^{2}\right)^{2 v} \otimes^{s} B^{t}(z, z)\left(F(z)-F_{r}(z)\right), F(z)-F_{r}(z)\right\rangle<\frac{\varepsilon^{2}}{4} .
\end{aligned}
$$

Now, take $r^{\prime}$ such that $\max \left(r_{1}, r_{2}\right)<r^{\prime}<1$. The sum $\sum_{|i|=s} \sum_{m=0}^{\infty} p_{m}^{(i)}\left(r^{\prime} z\right) e_{1}^{i_{1}} \odot$ $\cdots \odot e_{d}^{i_{d}}$ converges uniformly to $F_{r^{\prime}}(z)$ on $\mathrm{B}$. Hence there exists a natural number $N$ such that

$$
\left\|F_{r^{\prime}}-P_{N}\right\|_{v, s, \infty}^{2} \leq \sup _{z \in \mathrm{B}}\left\langle F_{r^{\prime}}(z)-P_{N}(z), F_{r^{\prime}}(z)-P_{N}(z)\right\rangle<\frac{\varepsilon^{2}}{4}
$$

where $P_{N}(z)=\sum_{|i|=s} \sum_{m=0}^{N} p_{m}^{(i)}\left(r^{\prime} z\right) e_{1}^{i_{1}} \odot \cdots \odot e_{d}^{i_{d}}$. This yields

$$
\left\|F-P_{N}\right\|_{v, s, \infty} \leq\left\|F-F_{r^{\prime}}\right\|_{\nu, s, \infty}+\left\|F_{r^{\prime}}-P_{N}\right\|_{\nu, s, \infty}<\varepsilon
$$

which completes the proof of the lemma.

Now we can prove the sufficiency of Theorem 1.1(b).

Proof of Sufficiency. Let $\varepsilon>0$ be given. Then it follows from Lemma 6.2 that there is a $P_{N}$ such that $\left\|F-P_{N}\right\|_{\nu, s, \infty}<\varepsilon$. Then the bilinear Hankel form $H_{F-P_{N}}^{s}=H_{F}^{s}-H_{P_{N}}^{s}$ with $F-P_{N}$ is bounded. In fact, the operator norm $\|\cdot\|$ satisfies

$$
\left\|H_{F}^{s}-H_{P_{N}}^{s}\right\| \leq C\left\|F-P_{N}\right\|_{\nu, s, \infty}<C \varepsilon .
$$

If we can prove that $H_{P_{N}}^{s}$ is compact then we are done. Actually we shall find that $H_{P_{N}}^{s}$ is of Hilbert-Schmidt class $\mathscr{S}_{2}$ and thus especially compact. By construction (see Lemma 6.2) $P_{N}$ is a linear combination of terms $z^{\gamma^{\prime}} e^{\gamma}=$ $z^{\gamma^{\prime}} e_{1}^{\gamma_{1}} \odot \cdots \odot e_{d}^{\gamma_{d}}$ so it is enough to prove that $H_{z^{\gamma^{\prime}} e^{\gamma}}^{s} \in \mathscr{S}_{2}$. Consider

$$
H_{z^{\gamma^{\prime} e^{\gamma}}}^{s}\left(z^{\alpha}, z^{\beta}\right)=\int_{\mathbf{B}}\left\langle\otimes^{s} B^{t}(w, w) \mathscr{T}_{s}\left(z^{\alpha}, z^{\beta}\right)(w), w^{\gamma^{\prime}} e_{1}^{\gamma_{1}} \odot \cdots \odot e_{d}^{\gamma_{d}}\right\rangle d \iota_{2 v}(w) .
$$


First we observe that

$$
\left\langle\otimes^{s} B^{t}(w, w) \mathscr{T}_{s}\left(z^{\alpha}, z^{\beta}\right)(w), w^{\gamma^{\prime}} e_{1}^{\gamma_{1}} \odot \cdots \odot e_{d}^{\gamma_{d}}\right\rangle
$$

is a linear combination of terms

$$
\begin{array}{r}
\left\langle\otimes^{s} B^{t}(w, w)\left(\partial_{1}^{i_{1}} \cdots \partial_{d}^{i_{d}}\right)\left(w^{\alpha}\right)\left(\partial_{1}^{j_{1}} \cdots \partial_{d}^{j_{d}}\right)\left(w^{\beta}\right) u_{1} \otimes u_{2} \otimes \cdots \otimes u_{s}\right. \\
\left.w^{\gamma^{\prime}} v_{1} \otimes v_{2} \otimes \cdots \otimes v_{s}\right\rangle
\end{array}
$$

where $u_{1} \otimes \cdots \otimes u_{s}$ and $v_{1} \otimes \cdots \otimes v_{s}$ contains $i_{k}+j_{k}$ copies and $\gamma_{k}$ copies of $e_{k}$ respectively. We may assume that $\alpha_{k} \geq i_{k}$ and $\beta_{k} \geq j_{k}$ for $k=1,2, \ldots d$. Denote $i=\left(i_{1}, \ldots, i_{d}\right)$ and $j=\left(j_{1}, \ldots, j_{d}\right)$. Then the term (27) equals

$$
C_{i, j}\left(1-|w|^{2}\right)^{s} w^{(\alpha+\beta)-(i+j)} \bar{w}^{\gamma^{\prime}} \prod_{m=1}^{s}\left(\left\langle u_{m}, v_{m}\right\rangle-\left\langle u_{m}, \bar{w}\right\rangle\left\langle\bar{w}, v_{m}\right\rangle\right)
$$

But this term yields a nonzero integral only for those $\alpha$ and $\beta$ with $|\alpha+\beta| \leq$ $\left|\gamma^{\prime}\right|+s$. In fact, this proves that the form $H_{z^{\gamma^{\prime}} e^{\gamma}}^{s}$ has finite rank. Thus

$$
\left\|H_{z^{\gamma^{\prime}} e^{\gamma}}^{s}\right\|_{\mathscr{S}_{2}}^{2}=\sum_{\alpha, \beta} \frac{\left|H_{z^{\gamma^{\prime}} e^{\gamma}}^{s}\left(z^{\alpha}, z^{\beta}\right)\right|^{2}}{\left\|z^{\alpha}\right\|_{v}^{2}\left\|z^{\beta}\right\|_{v}^{2}}
$$

with a finite sum. Hence $H_{z^{\gamma^{\prime}} e^{\gamma}}^{s} \in \mathscr{S}_{2}$ so that $H_{P_{N}}^{s} \in \mathscr{S}_{2}$.

Now we prove the necessity of Theorem 1.1(b).

Proof of THE NeCESSITY. Let $F$ be a symbol such that $H_{F}^{s}$ is compact. Since $\bigodot^{s} V^{\prime}$ is a finite dimensional Hilbert space we need only to prove that $\left\langle u_{n}, v\right\rangle \rightarrow 0$ as $n \rightarrow \infty$ where

$$
u_{n}=\left(\left(1-\left|z_{n}\right|^{2}\right)^{2 v} \otimes^{s} B^{t}\left(z_{n}, z_{n}\right)\right)^{1 / 2} F\left(z_{n}\right)
$$

and $\left|z_{n}\right| \nearrow 1$ as $n \rightarrow \infty$, for any $v \in \odot^{s} V^{\prime}$. As in the proof of the necessity of Theorem 1.1 (a) we write

$$
v=\sum_{|i|=s} v_{i} e_{1}^{i_{1}} \odot e_{2}^{i_{2}} \odot \cdots \odot e_{d}^{i_{d}}
$$

and let

$$
f(w)=\sum_{|i|=s} w_{1}^{i_{1}} \cdots w_{d}^{i_{s}} \cdot v_{i} \quad \text { and } \quad g(w)=1 .
$$


So for any symbol $S$ we have

$$
|\langle S(0), v\rangle|=C_{v, s}\left|H_{S}^{s}(f, g)\right|,
$$

by the same arguments as for (22) in the proof of the necessity of Theorem 1.1(a). Let

$$
S(w)=\pi_{\nu, s}\left(\left(\varphi_{z_{n}}\right) F\right)(w) \otimes^{s} \varphi_{z_{n}}^{\prime}(w)^{t} F\left(\varphi_{z_{n}}(w)\right)\left(J_{\varphi_{z n}}(w)\right)^{2 v /(d+1)}
$$

so that

$$
S(0)=\otimes^{s} \varphi_{z_{n}}^{\prime}(0)^{t} F\left(z_{n}\right)\left(J_{\varphi_{z n}}(0)\right)^{2 v /(d+1)} .
$$

By Proposition 2.2,

$$
J_{\varphi_{z n}}(0)=(-1)^{d}\left(1-\left|z_{n}\right|^{2}\right)^{(d+1) / 2} \quad \text { and } \quad B^{t}\left(z_{n}, z_{n}\right)^{1 / 2}=-\varphi_{z_{n}}^{\prime}(0)^{t}
$$

so that

$$
|\langle S(0), v\rangle|=\left|\left\langle u_{n}, v\right\rangle\right| .
$$

On the other hand

$$
H_{S}^{s}(f, g)=H_{F}^{s}\left(f \circ \varphi_{z_{n}} \cdot J_{\varphi_{z_{n}}}^{\nu /(d+1)}, k_{z_{n}}\right)
$$

where

$$
k_{z_{n}}(w)=\left(g \circ \varphi_{z_{n}}\right)(w)\left(J_{\varphi_{z_{n}}}(w)\right)^{v /(d+1)}=\rho \cdot \frac{\left(1-\left|z_{n}\right|^{2}\right)^{v / 2}}{\left(1-\left\langle w, z_{n}\right\rangle\right)^{v}}, \quad|\rho|=1,
$$

so that $k_{z_{n}}(w) \rightarrow 0$ weakly as $n \rightarrow \infty$ and $\left\|k_{z_{n}}\right\|_{\nu}=1$. Since $H_{F}^{s}$ is compact then there is a sequence $\left\{c_{n}\right\}_{n=0}^{\infty}$ of positive numbers such that $c_{n} \rightarrow 0$ and

$$
\left|H_{F}^{s}\left(h, k_{z_{n}}\right)\right| \leq c_{n}\|h\|_{\nu}
$$

for all $h \in L_{a}^{2}\left(d \iota_{\nu}\right)$. Let $h=f \circ \varphi_{z_{n}} \cdot J_{\varphi_{z n}}^{\nu /(d+1)}=\pi_{\nu}\left(\varphi_{z_{n}}\right) f$ which yields

$$
\|h\|_{v}^{2}=\|f\|_{v}^{2} .
$$

Then

$$
\left|\left\langle u_{n}, v\right\rangle\right| \leq C_{v, s} c_{n}\|f\|_{v} \leq C_{v, s}^{\prime} c_{n}\|v\|
$$

so that $\left\langle u_{n}, v\right\rangle \rightarrow 0$ as $n \rightarrow \infty$, which, combined with the equalities (28) and (29), implies that

$$
\left\langle\left(1-\left|z_{n}\right|^{2}\right)^{2 v} \otimes^{s} B^{t}\left(z_{n}, z_{n}\right) F\left(z_{n}\right), F\left(z_{n}\right)\right\rangle \rightarrow 0 \quad \text { as } \quad\left|z_{n}\right| \nearrow 1 .
$$




\subsection{Hilbert-Schmidt properties}

In this subsection we prove Theorem 1.2. Denote by $\mathscr{H}_{\nu, s}^{\prime}$ the space of all holomorphic functions $F: \mathrm{B} \rightarrow \odot^{s} V^{\prime}$ such that the corresponding bilinear Hankel form on $L_{a}^{2}\left(d \iota_{v}\right) \otimes L_{a}^{2}\left(d \iota_{v}\right)$

$$
H_{F}^{s}(f, g)=\int_{\mathrm{B}}\left\langle\otimes^{s} B^{t}(z, z) \mathscr{T}_{s}(f, g)(z), F(z)\right\rangle d \iota_{2 v}(z)
$$

is of Hilbert-Schmidt class $\mathscr{T}_{2}$. By Lemma 6.5 , it is a Hilbert space with an inner product $\langle F, S\rangle_{\nu, s}^{\prime}=\left\langle H_{F}^{s}, H_{S}^{s}\right\rangle_{\mathscr{S}_{2}}$ where

$$
\left\langle H_{F}^{s}, H_{S}^{s}\right\rangle_{\mathscr{S}_{2}}=\sum_{|\alpha|=0}^{\infty} \sum_{|\beta|=0}^{\infty} H_{F}^{s}\left(e_{\alpha}, e_{\beta}\right) \overline{H_{S}^{s}\left(e_{\alpha}, e_{\beta}\right)}
$$

and $e_{\alpha}=z^{\alpha} /\left\|z^{\alpha}\right\|_{\nu}$.

Lemma 6.5. The space $\mathscr{H}_{v, s}^{\prime}$ is a Hilbert space.

Proof. Let $\left\{F_{n}\right\}_{n=0}^{\infty}$ be a Cauchy sequence in $\mathscr{H}_{v, s}^{\prime}$. Then $\left\{H_{F_{n}}^{s}\right\}_{n=0}^{\infty}$ is Cauchy in operator norm so that $\left\{F_{n}\right\}_{n=0}^{\infty}$ is Cauchy in $\|\cdot\|_{\nu, s, \infty}$. Then there is a $F \in \mathscr{H}_{\nu, s}^{\infty}$ such that $F_{n} \rightarrow F$ in $\|\cdot\|_{v, s, \infty}$. Thus $H_{F_{n}}^{s} \rightarrow H_{F}^{s}$ in operator norm. On the other hand, the space of all bilinear forms of Hilbert-Schmidt class $\mathscr{S}_{2}$ is a Hilbert space so that $H_{F_{n}}^{s} \rightarrow H \in \mathscr{S}_{2}$ in $\|\cdot\|_{\mathscr{S}_{2}}$. Then $H_{F_{n}}^{s} \rightarrow H$ in operator norm so that $H=H_{F}^{s}$. Thus $F \in \mathscr{H}_{v, s}^{\prime}$ and $F_{n} \rightarrow F$ in $\mathscr{H}_{v, s}^{\prime}$.

We now shall see that $\mathscr{H}_{v, s}^{\prime}=\mathscr{H}_{v, s}^{2}$, namely they are equal as sets and the norms are equivalent, as is shown below. Actually, Theorem 1.2 is a direct consequence of Theorem 6.6.

THEOREM 6.6. There is a constant $C_{v, s}>0$ such that

$$
\|F\|_{\nu, s}^{\prime}=C_{\nu, s}\|F\|_{\nu, s, 2}
$$

for all holomorphic $F: \mathrm{B} \rightarrow \odot^{s} V^{\prime}$.

To prove Theorem 6.6 we need some lemmas.

Lemma 6.7. Let $\left\{e_{1}, \ldots, e_{d}\right\}$ be an orthonormal basis for $V^{\prime}$. Then the spaces $\mathscr{H}_{v, s}^{\prime}$ and $\mathscr{H}_{v, s}^{2}$ contains the element $e_{1}^{s}=e_{1} \otimes \cdots \otimes e_{1}$.

Proof. Clearly $e_{1}^{s} \in \mathscr{H}_{\nu, s}^{2}$. The fact that $e_{1}^{s} \in \mathscr{H}_{\nu, s}^{\prime}$ follows from (27), letting $\gamma^{\prime}=0$ and $\gamma_{j}=s \cdot \delta_{1 j}$ for $j=1, \ldots, d$.

Lemma 6.8. The action $\pi_{v, s}$, defined in (15), is unitary on both $\mathscr{H}_{v, s}^{\prime}$ and $\mathscr{H}_{v, s}^{2}$. 
Proof. Clearly, $\pi_{v, s}$ is unitary on $\mathscr{H}_{v, s}^{2}$. That $\pi_{v, s}$ is also unitary on $\mathscr{H}_{v, s}^{\prime}$ follows from Lemma 3.2 and the fact that $\pi_{v}$, defined in (13), is unitary on $L_{a}^{2}\left(d \iota_{v}\right)$.

Lemma 6.9. The space $\mathscr{H}_{v, s}^{2}$ is irreducible with respect to the action $\pi_{v, s}$, defined in (15).

Proof. Let $\mathscr{H}_{0} \subset \mathscr{H}_{v . s}^{2}$ be invariant under the action $\pi_{v, s}(g), g \in G$, and assume that $h \in \mathscr{H}_{0}$ for some $h \neq 0$. We may assume, by replacing $h$ by an action of $\pi_{v, s}(g)$ on $h$ if necessary, that $h(0) \neq 0$. We need to prove

$$
f \in \mathscr{H}_{v, s}^{2}, f \perp \mathscr{H}_{0} \Longrightarrow f=0 .
$$

Take such an $f \in \mathscr{H}_{\nu, s}^{2}$. Since $e^{i \theta}: z \rightarrow e^{i \theta} z$ is in $G$ and

$$
\mathscr{H}_{0} \ni\left(\pi_{\nu, s}\left(e^{i \theta}\right) h\right)(z)=\left(e^{-i \theta d}\right)^{2 v /(d+1)} \cdot e^{-i \theta s} \cdot h\left(e^{i \theta} z\right)
$$

then $h\left(e^{i \theta} z\right) \in \mathscr{H}_{0}$. Hence, by the mean value property,

$$
h(0)=\int_{0}^{2 \pi} h\left(e^{i \theta} z\right) d \theta \in \mathscr{H}_{0} .
$$

Then we have found a nonzero element in $\odot^{s} V^{\prime}$ which is also contained in $\mathscr{H}_{0}$. Then $v \in \mathscr{H}_{0}$ for any $v \in \odot^{s} V^{\prime}$ (by Theorem 12.2.8 in [20]). Then $\left[\pi_{v, s}\left(\varphi_{w}\right) v\right](z)=c \cdot K(z, w) v$ is in $\mathscr{H}_{0}$, for any $v \in \odot^{s} V^{\prime}$, where $K(\cdot, w)$ is the reproducing kernel for $\mathscr{H}_{v, s}^{2}$ and $c$ is a nonzero constant. Hence

$$
f \perp K(\cdot, w) v
$$

so that

$$
f(w)=0 \quad \text { for all } \quad w \in \mathbf{B}
$$

by the reproducing property. This proves (30).

Now we can prove Theorem 6.6.

Proof of Theorem 6.6. As a consequence of Theorem VI.23 in [16] we can make the following identification of the space $\mathscr{S}_{2}\left(L_{a}^{2}\left(d \iota_{v}\right), L_{a}^{2}\left(d \iota_{v}\right)\right)$ of Hilbert-Schmidt bilinear forms on $L_{a}^{2}\left(d \iota_{v}\right)$ with the tensor product, that is,

$$
\mathscr{T}_{2}\left(L_{a}^{2}\left(d \iota_{v}\right), L_{a}^{2}\left(d \iota_{v}\right)\right)=L_{a}^{2}\left(d \iota_{v}\right) \otimes L_{a}^{2}\left(d \iota_{v}\right) .
$$

Moreover $L_{a}^{2}\left(d \iota_{v}\right) \otimes L_{a}^{2}\left(d \iota_{v}\right)$ can be decomposed into irreducible subspaces $\tilde{\mathscr{H}}_{v, s}$ of Hankel forms of weight $s$ with an intertwining operator $T: \mathscr{H}_{v, s}^{2} \rightarrow$ $\tilde{\mathscr{H}}_{v, s}$, (see [15]). Also, $H_{F}^{s}$ defined in (7) is a Hankel form of weight $s$ and by 
Lemma 6.7 there is a nonzero element in $\mathscr{H}_{v, S}^{2}$ which yields a nonzero element in $\mathscr{H}_{v, s}^{\prime}$. Thus

$$
\mathscr{H}_{v, s}^{\prime}=\mathscr{H}_{v, s}^{2}
$$

whose norms are the same up to a constant, by Corollary 8.13 in [9].

\section{Matrix-valued Bergman type projections}

To prove Theorem 1.3 we need certain interpolation results for the spaces $\mathscr{H}_{\nu, S}^{p}$, which will then be derived from certain $L^{p}$-boundedness properties of some matrix-valued Bergman projections. The results in this section might be of independent interests. We refer to Zhu [22] for the study of boundedness property of scalar Bergman projections.

We start with a technical lemma.

Lemma 7.1. Let s be a positive integer. Then

$$
\begin{aligned}
\| \otimes^{s} B^{t}(w, w)^{1 / 2} \otimes^{s}\left(B^{t}(w, z)\right)^{-1} \otimes^{s} & B^{t}(z, z)^{1 / 2} \| \\
& \leq C_{s} \cdot \frac{\left(1-|w|^{2}\right)^{s / 2}\left(1-|z|^{2}\right)^{s / 2}}{|1-\langle w, z\rangle|^{s}}
\end{aligned}
$$

for all $w, z \in \mathbf{B}$.

Proof. First we shall prove the lemma for $s=1$ by using the following identities (see (10) and (4)):

$$
B^{t}(z, z)^{1 / 2}=s_{z}\left(s_{z} P_{\bar{z}}+Q_{\bar{z}}\right) \quad \text { where } \quad s_{z}=\left(1-|z|^{2}\right)^{1 / 2}
$$

and

$$
B^{t}(w, z)^{-1}=(1-\langle w, z\rangle)^{-2}\left((1-\langle w, z\rangle) I+\bar{z} \otimes \bar{w}^{*}\right) .
$$

Note that

$$
\begin{aligned}
B^{t}(w, w)^{1 / 2} & B^{t}(w, z)^{-1} B^{t}(z, z)^{1 / 2} \\
= & s_{w} s_{z}(1-\langle w, z\rangle)^{-1}\left(s_{w} P_{\bar{w}}+Q_{\bar{w}}\right)\left(s_{z} P_{\bar{z}}+Q_{\bar{z}}\right) \\
& +s_{w} s_{z}(1-\langle w, z\rangle)^{-2}\left(s_{w} P_{\bar{w}}+Q_{\bar{w}}\right)\left(\bar{z} \otimes \bar{w}^{*}\right)\left(s_{z} P_{\bar{z}}+Q_{\bar{z}}\right) .
\end{aligned}
$$

Thus, by the inequality

$$
\frac{\left(1-|z|^{2}\right)\left(1-|w|^{2}\right)}{|1-\langle w, z\rangle|^{2}} \leq 1 \quad \text { for all } \quad z, w \in \mathrm{B},
$$

it is enough to show that

$$
\left\|\left(s_{w} P_{\bar{w}}+Q_{\bar{w}}\right)\left(\bar{z} \otimes \bar{w}^{*}\right)\left(s_{z} P_{\bar{z}}+Q_{\bar{z}}\right)\right\| \leq C|1-\langle w, z\rangle| .
$$


To this end, we may assume $|z| \geq 1 / 2$ and $|w| \geq 1 / 2$. Expand the product $(\cdot)(\cdot)(\cdot)$ as a sum of four terms. First we note that

$$
\left\|s_{w} P_{\bar{w}}\left(\bar{z} \otimes \bar{w}^{*}\right) s_{z} P_{\bar{z}}\right\| \leq s_{w} s_{z} \leq|1-\langle w, z\rangle| .
$$

There are three parts left to consider. Let $v \in V^{\prime}$. The first part to estimate is

$$
s_{w} P_{\bar{w}}\left(\bar{z} \otimes \bar{w}^{*}\right) Q_{\bar{z}} v=s_{w}\left\langle v, \bar{w}-\frac{\langle z, w\rangle}{|z|^{2}} \bar{z}\right\rangle \frac{\langle z, w\rangle}{|w|^{2}} \bar{w} .
$$

We use Cauchy-Schwarz' inequality. Note that

$$
\left\|\bar{w}-\frac{\langle z, w\rangle}{|z|^{2}} \bar{z}\right\|^{2}=\frac{|w|^{2}|z|^{2}-|\langle z, w\rangle|^{2}}{|z|^{2}} \leq 8|1-\langle z, w\rangle| .
$$

Thus the inequalities $1-|w|^{2} \leq 2|1-\langle z, w\rangle|$ and $|w| \geq 1 / 2$ yield the estimation

$$
\left\|s_{w} P_{\bar{w}}\left(\bar{z} \otimes \bar{w}^{*}\right) Q_{\bar{z}} v\right\| \leq 16|1-\langle z, w\rangle|\|v\|
$$

Since

$$
Q_{\bar{w}}\left(\bar{z} \otimes \bar{w}^{*}\right) s_{z} P_{\bar{z}}=\left(s_{z} P_{\bar{z}}\left(\bar{w} \otimes \bar{z}^{*}\right) Q_{\bar{w}}\right)^{*}
$$

we have an estimation of the second part

$$
\left\|Q_{\bar{w}}\left(\bar{z} \otimes \bar{w}^{*}\right) s_{z} P_{\bar{z}} v\right\| \leq 16|1-\langle z, w\rangle|\|v\| .
$$

Finally consider

$$
Q_{\bar{w}}\left(\bar{z} \otimes \bar{w}^{*}\right) Q_{\bar{z}} v=\left\langle v, \bar{w}-\frac{\langle z, w\rangle}{|z|^{2}} \bar{z}\right\rangle\left(\bar{z}-\frac{\langle w, z\rangle}{|w|^{2}} \bar{w}\right) .
$$

The same estimates as above yield

$$
\left\|Q_{\bar{w}}\left(\bar{z} \otimes \bar{w}^{*}\right) Q_{\bar{z}} v\right\| \leq 8|1-\langle z, w\rangle|\|v\| .
$$

Thus the four estimations (32), (33), (34) and (35) yields (31). We have proved the lemma for $s=1$. Now, consider the case where $s=2,3, \ldots$ and let

$$
A_{w, z}=B^{t}(w, w)^{1 / 2}\left(B^{t}(w, z)\right)^{-1} B^{t}(z, z)^{1 / 2}
$$

and

$$
t_{w, z}=\frac{s_{z} s_{w}}{|1-\langle z, w\rangle|} .
$$

We have proved that

$$
A_{w, z}^{*} A_{w, z} \leq C^{2} t_{w, z}^{2} I
$$


so that

$$
\left(\otimes^{s} A_{w, z}\right)^{*} \otimes^{s} A_{w, z}=\otimes^{s}\left(A_{w, z}^{*} A_{w, z}\right) \leq C^{2 s} t_{w, z}^{2 s} \otimes^{s} I
$$

which proves the lemma.

THEOREM 7.2. Let $\alpha>d$ and let $P_{v, s}: L_{v, s}^{2} \rightarrow \mathscr{H}_{v, s}^{2}$ be the orthogonal projection operator. If $\max \{(\alpha-d) /(2 v+s / 2-d), 1\}<p<\infty$, then

$$
\begin{aligned}
\int_{\mathrm{B}}\left\|\otimes^{s} B^{t}(z, z)^{1 / 2} P_{\nu, s} f(z)\right\|^{p} & \left(1-|z|^{2}\right)^{\alpha} d \iota(z) \\
\leq & C \int_{\mathrm{B}}\left\|\otimes^{s} B^{t}(w, w)^{1 / 2} f(w)\right\|^{p}\left(1-|w|^{2}\right)^{\alpha} d \iota(w) .
\end{aligned}
$$

Remark 7.3. By Lemma 3.5 the orthogonal projection operator $P_{v, s}$, such that for any $f \in L_{v, s}^{2}$ and any $v \in \odot^{s} V^{\prime}$ we have that (36)

$$
\left\langle P_{v, s} f(z), v\right\rangle=c \int_{\mathrm{B}}\left\langle\left(1-|w|^{2}\right)^{2 v} \otimes^{s} B^{t}(w, w) f(w), K_{v, s}(w, z) v\right\rangle d \iota(w)
$$

where

$$
K_{v, s}(w, z)=\otimes^{s}\left(B^{t}(w, z)\right)^{-1}(1-\langle w, z\rangle)^{-2 v},
$$

is well-defined.

Proof of Theorem 7.2. The formula (36) can be rewritten as

$$
P_{v, s} f(z)=c \int_{\mathrm{B}} K_{v, s}(w, z)^{*} \otimes^{s} B^{t}(w, w) f(w)\left(1-|w|^{2}\right)^{2 v} d \iota(w) .
$$

Now let

$$
T(z, w)=\frac{\left(1-|z|^{2}\right)^{s / 2}\left(1-|w|^{2}\right)^{2 v+s / 2-\alpha}}{|1-\langle z, w\rangle|^{2 v+s}} .
$$

By the equality $K_{v, s}(w, z)^{*}=K_{v, s}(z, w)$ and Lemma 7.1 it follows that

$$
\begin{aligned}
\| \otimes^{s} B^{t}(z, z)^{1 / 2} & P_{v, s} f(z) \| \\
& \leq C \int_{\mathrm{B}} T(z, w)\left\|\otimes^{s} B^{t}(w, w)^{1 / 2} f(w)\right\|\left(1-|w|^{2}\right)^{\alpha} d \iota(w) .
\end{aligned}
$$

We claim that there exists a real number $t$ such that

$$
\int_{\mathrm{B}} T(z, w)\left(1-|w|^{2}\right)^{q t}\left(1-|w|^{2}\right)^{\alpha} d \iota(w) \leq M\left(1-|z|^{2}\right)^{q t}
$$

and

$$
\int_{\mathrm{B}} T(z, w)\left(1-|z|^{2}\right)^{p t}\left(1-|z|^{2}\right)^{\alpha} d \iota(z) \leq M\left(1-|w|^{2}\right)^{p t}
$$


holds for some constant $M$, where $q$ is given by $1=1 / p+1 / q$. Accepting temporarily the claim, using Hölder's inequality and (37),

$$
\begin{aligned}
\left\|\otimes^{s} B^{t}(z, z)^{1 / 2} P_{v, s} f(z)\right\| & \\
\leq & C\left(\int_{\mathrm{B}} T(z, w)\left(1-|w|^{2}\right)^{q t}\left(1-|w|^{2}\right)^{\alpha} d \iota(w)\right)^{1 / q} . \\
& \left(\int_{\mathrm{B}} T(z, w)\left(1-|w|^{2}\right)^{-p t}\left\|\otimes^{s} B^{t}(w, w)^{1 / 2} f(w)\right\|^{p}\left(1-|w|^{2}\right)^{\alpha} d \iota(w)\right)^{1 / p} \\
\leq & C M^{1 / q}\left(1-|z|^{2}\right)^{t} . \\
& \left(\int_{\mathrm{B}} T(z, w)\left(1-|w|^{2}\right)^{-p t}\left\|\otimes^{s} B^{t}(w, w)^{1 / 2} f(w)\right\|^{p}\left(1-|w|^{2}\right)^{\alpha} d \iota(w)\right)^{1 / p} .
\end{aligned}
$$

Thus, by Fubini-Tonelli's theorem and (38), we have that

$$
\begin{aligned}
& \int_{\mathrm{B}}\left\|\otimes^{s} B^{t}(z, z)^{1 / 2} P_{v, s} f(z)\right\|^{p}\left(1-|z|^{2}\right)^{\alpha} d \iota(z) \\
& \leq C^{p} M^{p / q} \int_{\mathrm{B}}\left(1-|z|^{2}\right)^{p t}\left(\int_{\mathrm{B}} T(z, w)\left(1-|w|^{2}\right)^{-p t}\left\|\otimes^{s} B^{t}(w, w)^{1 / 2} f(w)\right\|^{p} .\right. \\
& \left.\quad\left(1-|w|^{2}\right)^{\alpha} d \iota(w)\right)\left(1-|z|^{2}\right)^{\alpha} d \iota(z) \\
& =C^{p} M^{p / q} \int_{\mathrm{B}}\left(1-|w|^{2}\right)^{-p t}\left\|\otimes^{s} B^{t}(w, w)^{1 / 2} f(w)\right\|^{p}\left(1-|w|^{2}\right)^{\alpha} . \\
& \qquad\left(\int_{\mathrm{B}}\left(1-|z|^{2}\right)^{p t} T(z, w)\left(1-|z|^{2}\right)^{\alpha} d \iota(z)\right) d \iota(w) \\
& \leq C^{p} M^{p} \int_{\mathrm{B}}\left\|\otimes^{s} B^{t}(w, w)^{1 / 2} f(w)\right\|^{p}\left(1-|w|^{2}\right)^{\alpha} d \iota(z),
\end{aligned}
$$

namely our theorem.

Now we go back to (37) and (38) which, by Lemma 2.4, holds if

$$
\frac{d-2 v-s / 2}{q}<t<\frac{s}{2 q}
$$

and

$$
\frac{d-s / 2-\alpha}{p}<t<\frac{2 v+s / 2-\alpha}{p}
$$

respectively. Actually, by simple computations,

$$
\left(\frac{d-2 v-s / 2}{q}, \frac{s}{2 q}\right) \bigcap\left(\frac{d-s / 2-\alpha}{p}, \frac{2 v+s / 2-\alpha}{p}\right) \neq \emptyset
$$


if $\max \{(\alpha-d) /(2 v+s / 2-d), 1\}<p<\infty$.

Corollary 7.4. If $1<p<\infty$, then

$$
P_{\nu, s} L_{v, s}^{p}=\mathscr{H}_{v, s}^{p},
$$

namely $P_{\nu, s}: L_{v, s}^{p} \rightarrow \mathscr{H}_{v, s}^{p}$ is bounded.

\section{Application of the boundedness of $\boldsymbol{P}_{\boldsymbol{v}, s}$}

\subsection{Some interpolation results}

In this subsection we use the complex interpolation method of Banach spaces to prove Theorem 8.2, which we will use to prove Theorem 1.3 in subsection 8.2.

The spaces $\mathscr{A}_{1}=L_{v, s}^{2}+L_{v, s}^{\infty}$ and $\mathscr{A}_{2}=\mathscr{H}_{\nu, s}^{2}+\mathscr{H}_{v, s}^{\infty}$ are Banach spaces with the norms

$$
\|F\|_{\mathscr{A}_{i}}=\inf \left\{\left\|F_{2}\right\|_{v, s, 2}+\left\|F_{\infty}\right\|_{\nu, s, \infty}: F=F_{2}+F_{\infty} \in \mathscr{A}_{i}\right\},
$$

$i=1,2$, respectively, by Lemma 2.3.1 in [1]. Denote by $\mathscr{F}_{i}=\mathscr{F}_{(}\left(\mathscr{A}_{i}\right)$, $i=1,2$, the space of all functions with values in $\mathscr{A}_{i}$, which are bounded and continuous on the strip

$$
S=\{z \in \mathrm{C}: 0 \leq \Re z \leq 1\}
$$

and holomorphic on the open strip

$$
S_{0}=\{z \in \mathrm{C}: 0<\Re z<1\}
$$

and moreover, the functions $t \rightarrow f(j+i t)$ are continuous functions from the real line such that $f(i t) \in L_{v, s}^{2}\left(\right.$ resp. $\left.\mathscr{H}_{v, s}^{2}\right)$ and $f(1+i t) \in L_{v, s}^{\infty}$ (resp. $\mathscr{H}_{v, s}^{\infty}$ ), which tends to zero as $|t| \rightarrow \infty$. Then $\mathscr{F}_{i}, i=1,2$, are Banach spaces with the same norm

$$
\|f\|_{\mathscr{F}}=\max \left(\sup \|f(i t)\|_{\nu, s, 2}, \sup \|f(1+i t)\|_{\nu, s, \infty}\right),
$$

by Lemma 4.1.1 in [1]. Now let $0<\theta<1$ and denote by $\left(L_{\nu, s}^{2}, L_{v, s}^{\infty}\right)_{[\theta]}$ and $\left(\mathscr{H}_{\nu, s}^{2}, \mathscr{H}_{\nu, S}^{\infty}\right)_{[\theta]}$ the space of all $S \in \mathscr{A}_{i}$ such that

$$
\|S\|_{i,[\theta]}=\inf \left\{\|f\|_{\mathscr{F}}: f(\theta)=S, f \in \mathscr{F}_{i}\right\}<\infty,
$$

$i=1,2$, respectively.

Lemma 8.1. If $2<p<\infty$, then

$$
P_{v, s}\left(L_{v, s}^{2}, L_{v, s}^{\infty}\right)_{[1-2 / p]}=\left(\mathscr{H}_{v, s}^{2}, \mathscr{H}_{v, s}^{\infty}\right)_{[1-2 / p]},
$$


namely $P_{v, s}:\left(L_{v, s}^{2}, L_{\nu, s}^{\infty}\right)_{[1-2 / p]} \rightarrow\left(\mathscr{H}_{\nu, s}^{2}, \mathscr{H}_{\nu, s}^{\infty}\right)_{[1-2 / p]}$ is bounded.

Proof. As a direct consequence of Lemma 7.1 we have that $P_{v, s}: L_{v, s}^{\infty} \rightarrow$ $\mathscr{H}_{\nu, s}^{\infty}$ is bounded. Indeed, for any $f \in L_{\nu, s}^{\infty}$,

$$
\begin{aligned}
\| \otimes^{s} & B^{t}(z, z)^{1 / 2} P_{\nu, s} f(z) \| \\
& \leq C\left(1-|z|^{2}\right)^{s / 2} \int_{\mathrm{B}}\left\|\otimes^{s} B^{t}(w, w)^{1 / 2} f(w)\right\| \cdot \frac{\left(1-|w|^{2}\right)^{2 v+s / 2}}{|1-\langle w, z\rangle|^{2 v}} d \iota(w) \\
& \leq C\left(1-|z|^{2}\right)^{s / 2}\|f\|_{\nu, s, \infty} \int_{\mathrm{B}} \frac{\left(1-|w|^{2}\right)^{\nu+s / 2}}{|1-\langle w, z\rangle|^{2 v}} d \iota(w) \\
& \leq C^{\prime}\|f\|_{\nu, s, \infty}\left(1-|z|^{2}\right)^{-v}
\end{aligned}
$$

where the last inequality follows from Lemma 2.4. Hence, the result follows from Riesz-Thorin's interpolation theorem.

If we claim that

$$
\left(L_{v, s}^{2}, L_{v, s}^{\infty}\right)_{[1-2 / p]}=L_{v, s}^{p}, \quad 2<p<\infty,
$$

then we have the following theorem.

THEOREM 8.2. If $2<p<\infty$, then

$$
\mathscr{H}_{v, s}^{p}=\left(\mathscr{H}_{v, s}^{2}, \mathscr{H}_{v, s}^{\infty}\right)_{[1-2 / p]} .
$$

Proof. If $2<p<\infty$, then by the identity (41) we have that

$$
L_{v, s}^{p}=\left(L_{\nu, s}^{2}, L_{\nu, s}^{\infty}\right)_{[1-2 / p]} .
$$

Thus, by Corollary 7.4 and Lemma 8.1, if $2<p<\infty$ then

$$
\mathscr{H}_{v, s}^{p}=P_{\nu, s} L_{v, s}^{p}=P_{\nu, s}\left(L_{v, s}^{2}, L_{v, s}^{\infty}\right)_{[1-2 / p]}=\left(\mathscr{H}_{v, s}^{2}, \mathscr{H}_{\nu, s}^{\infty}\right)_{[1-2 / p]} .
$$

The identity (41) can be proved by slightly modifying Theorem 5.1.1 in [1] using

(42) $\|F\|_{\nu, s, p}=\sup \left\{\left|\int_{B}\left\langle\left(1-|z|^{2}\right)^{2 v} \otimes^{s} B^{t}(z, z) F(z), S(z)\right\rangle d \iota(z)\right|\right.$ :

$S$ bounded with compact support, $\left.\|S\|_{\nu, s, q}=1\right\}$ 
where $1 / p+1 / q=1$. Indeed, to prove (42) let $F: \mathrm{B} \rightarrow \odot^{s} V^{\prime}$ be measurable. Then

$$
H=\left(\left(1-|\cdot|^{2}\right)^{2 v} \otimes^{s} B^{t}(\cdot, \cdot)\right)^{1 / 2} F: \mathrm{B} \rightarrow \odot^{s} V^{\prime}
$$

is measurable and we may write $H=\left(H_{1}, \ldots, H_{N}\right)$, where $\operatorname{dim}\left(\odot^{s} V^{\prime}\right)=N$. For $1 \leq j \leq N$ we can find bounded functions $b_{n}^{j}$ with compact support in $\mathrm{B}$ such that $\left|b_{n}^{j}\right| \nearrow\left|H_{j}\right|$. Let

$$
s_{n}^{j}=\left|b_{n}^{j}\right| \cdot e^{i \operatorname{Arg} H_{j}} .
$$

Then $s_{n}^{j}$ are bounded with compact support and

$$
H_{j} \cdot \overline{s_{n}^{j}}=\left|H_{j}\right| \cdot\left|b_{n}^{j}\right| .
$$

Let $s_{n}=\left(s_{1}^{1}, \ldots, s_{n}^{N}\right)$ and put

$$
t_{n}(z)=\left(\left(1-|z|^{2}\right)^{2 v} \otimes^{s} B^{t}(z, z)\right)^{-1 / 2} s_{n}(z) .
$$

Then $t_{n}: \mathrm{B} \rightarrow \odot^{s} V^{\prime}$ is measurable and

$$
\begin{aligned}
& \left\langle\left(1-|z|^{2}\right)^{2 v} \otimes^{s} B^{t}(z, z) t_{n}(z), t_{n}(z)\right\rangle \\
& \quad=\sum_{j=1}^{N} s_{n}^{j}(z) \cdot \overline{s_{n}^{j}(z)}=\sum_{j=1}^{N}\left|b_{n}^{j}(z)\right| \cdot\left|b_{n}^{j}(z)\right| \leq \sum_{j=1}^{N}\left|H_{j}(z)\right| \cdot\left|b_{n}^{j}(z)\right| \\
& \quad=\sum_{j=1}^{N} H_{j}(z) \cdot \overline{s_{n}^{j}(z)}=\left\langle\left(1-|z|^{2}\right)^{2 v} \otimes^{s} B^{t}(z, z) F(z), t_{n}(z)\right\rangle .
\end{aligned}
$$

Now, let

$$
S_{n}(z)=\frac{\left\langle\left(1-|z|^{2}\right)^{2 v} \otimes^{s} B^{t}(z, z) t_{n}(z),\left.t_{n}(z)\right|^{(q-2) / 2} \cdot t_{n}(z)\right.}{\left\|t_{n}\right\|_{v, s, q}^{q-1}} .
$$

Then $S_{n}: \mathrm{B} \rightarrow \odot^{s} V^{\prime}$ is measurable,

$$
\left\|S_{n}\right\|_{\nu, s, q}=1
$$

and

$$
\int_{\mathrm{B}}\left\langle\left(1-|z|^{2}\right)^{2 v} \otimes^{s} B^{t}(z, z) S_{n}(z), t_{n}(z)\right\rangle d \iota(z)=\left\|t_{n}\right\|_{v, s, p}
$$


so by (43)

$$
\begin{aligned}
\|F\|_{v, s, p} & \leq \underline{\lim }\left\|t_{n}\right\|_{v, s, p}=\underline{\lim } \int_{\mathrm{B}}\left\langle\left(1-|z|^{2}\right)^{2 v} \otimes^{s} B^{t}(z, z) S_{n}(z), t_{n}(z)\right\rangle d \iota(z) \\
& \leq \underline{\lim } \int_{\mathrm{B}}\left\langle\left(1-|z|^{2}\right)^{2 v} \otimes^{s} B^{t}(z, z) S_{n}(z), F(z)\right\rangle d \iota(z) \leq M_{p}(F)
\end{aligned}
$$

where

$$
\begin{array}{r}
M_{p}(F)=\sup \left\{\left|\int_{\mathrm{B}}\left\langle\left(1-|z|^{2}\right)^{2 v} \otimes^{s} B^{t}(z, z) F(z), S(z)\right\rangle d \iota(z)\right|:\right. \\
\left.\quad S \text { bounded with compact support, }\|S\|_{v, s, q}=1\right\} .
\end{array}
$$

On the other hand

$$
\left|\int_{\mathrm{B}}\left\langle\left(1-|z|^{2}\right)^{2 v} \otimes^{s} B^{t}(z, z) F(z), S(z)\right\rangle d \iota(z)\right| \leq\|F\|_{v, s, p} \cdot\|S\|_{v, s, q}
$$

which proves (42). The rest is almost the same as in [1] loc. cit., only replacing the usual absolute value $|g(z)|$ of scalar functions $g(z)$ by the norm $\|S(z)\|_{z}=\left\|\left(\left(1-|z|^{2}\right)^{2 v} \otimes^{s} B^{t}(z, z)\right)^{1 / 2} S(z)\right\|$ of vector-valued functions $S(z)$, also $E(z)=\langle f(z), g(z)\rangle$ by

$$
H(z)=\int_{B}\left\langle\left(1-|z|^{2}\right)^{2 v} \otimes^{s} B^{t}(z, z) F(z), \overline{S(z)}\right\rangle d \iota(z) .
$$

\subsection{Schatten-von Neumann properties}

In this subsection we prove Theorem 1.3.

ProOf OF SUFFICIENCY OF TheOREM 1.3. By Theorem 1.2 and Theorem 1.1 the operator $F \rightarrow H_{F}^{s}$ is bounded from $\mathscr{H}_{v, s}^{2}$ into $\mathscr{T}_{2}$ and from $\mathscr{H}_{v, s}^{\infty}$ into $\mathscr{S}_{\infty}$ respectively. Then it follows from Theorem 8.2 and Riesz-Thorin interpolation theorem that $F \rightarrow H_{F}^{s}$ is bounded from $\mathscr{H}_{v, s}^{p}$ into $\left(\mathscr{S}_{2}, \mathscr{S}_{\infty}\right)_{[1-2 / p]}$ if $2<p<$ $\infty$. By Theorem 2.10 in [21] we have that

$$
\mathscr{S}_{p}=\left(\mathscr{S}_{2}, \mathscr{S}_{\infty}\right)_{[1-2 / p]},
$$

so that the operator $F \rightarrow H_{F}^{s}$ is bounded from $\mathscr{H}_{v, s}^{p}$ into $\mathscr{S}_{p}$ if $2<p<\infty$.

The necessity of Theorem 1.3 is a direct consequence of Lemma 8.6 below. This Lemma states some boundedness properties for an operator $\tilde{\mathscr{T}}_{s}$ closely related to the Transvectant defined in (6) viewed as an operator from bilinear forms to vector-valued holomorphic functions, see also [4] and [15]. We need 
to construct $\tilde{\mathscr{T}}_{s}$. Let $A \in \mathscr{S}_{\infty}\left(L_{a}^{2}\left(d \iota_{v}\right), L_{a}^{2}\left(d \iota_{v}\right)\right)$. Then there is a conjugate linear operator $T: L_{a}^{2}\left(d \iota_{v}\right) \rightarrow \overline{L_{a}^{2}\left(d \iota_{v}\right)}$ such that

$$
A\left(K_{z}, K_{w}\right)=\left\langle K_{z}, T K_{w}\right\rangle_{v}=\left\langle K_{w}, T^{*} K_{z}\right\rangle_{v}=\overline{T^{*} K_{z}(w)} .
$$

Also,

$$
\begin{aligned}
A(f, g)=\langle f, T g\rangle_{v} & =\int_{\mathrm{B}} f(z) \overline{(T g)(z)} d \iota_{v}(z) \\
& =\int_{\mathrm{B}} f(z) \overline{\left\langle T g, K_{z}\right\rangle_{\nu}} d \iota_{v}(z) \\
& =\int_{\mathrm{B}} f(z) \overline{\left\langle T^{*} K_{z}, g\right\rangle_{v}} d \iota_{v}(z) \\
& =\int_{\mathrm{B}} \int_{\mathrm{B}} \overline{T^{*} K_{z}(w)} f(z) g(w) d \iota_{v}(z) d \iota_{v}(w) .
\end{aligned}
$$

Define $G(z, w):=G_{A}(z, w)=\overline{A\left(K_{z}, K_{w}\right)}$. Then $G(z, w)$ is holomorphic in $z$ and in $w$ and

$$
A(f, g)=\int_{\mathrm{B}} \int_{\mathrm{B}} \overline{G(z, w)} f(z) g(w) d \iota_{v}(z) d \iota_{v}(w) .
$$

Now, define

$$
\tilde{\mathscr{T}}_{s}(A)(z)=\left(\mathscr{T}_{s} G\right)(z, z)
$$

where

$$
\left(\mathscr{T}_{s} G\right)(z, w)=\sum_{k=0}^{s}(-1)^{s-k}\left(\begin{array}{l}
s \\
k
\end{array}\right) \frac{\partial_{z}^{k} \odot \partial_{w}^{s-k} G(z, w)}{(v)_{k}(\nu)_{s-k}} .
$$

REMARK 8.3. If $G(z, w)=f(z) g(w)$ where $f, g \in L_{a}^{2}\left(d \iota_{v}\right)$ then $\mathscr{T}_{s}(G)(z, z)=\mathscr{T}_{s}(f, g)(z)$ where $\mathscr{T}_{s}(f, g)(z)$ is the Transvectant defined in (6).

Lemma 8.4. Let $\tilde{\mathscr{T}}_{s}$ be defined on $\mathscr{S}_{\infty}$ as in (44). Then $\tilde{\mathscr{T}}_{s}: \mathscr{S}_{\infty} \rightarrow \mathscr{H}_{v, s}^{\infty}$ is bounded.

Proof. Let $A \in \mathscr{S}_{\infty}$. Let $G(z, w)=G_{A}(z, w)$. First we note that $\left(\mathscr{T}_{s} G\right)(z, w)$ is a linear combination of terms

$$
\partial_{z}^{k} \partial_{w}^{s-k} G(z, w)=\sum_{|I|=k,|J|=s-k} \partial_{z}^{I} \partial_{w}^{J} \overline{A\left(K_{z}, K_{w}\right)} d z_{I} \otimes d w_{J}
$$


where $i_{1}, \ldots, i_{k} \in\{1, \ldots, d\}, I=\left(i_{1}, \ldots, i_{k}\right), d z_{I}=d z_{i_{1}} \otimes \cdots \otimes d z_{i_{k}}$ and $\partial_{z}^{I}=\partial_{i_{1}} \partial_{i_{2}} \cdots \partial_{i_{k}}$. By the identity

$$
\partial_{i_{1}} \cdots \partial_{i_{k}} \partial_{j_{1}} \cdots \partial_{j_{s-k}} \overline{A\left(K_{z}, K_{w}\right)}=\overline{A\left(E_{z}, E_{w}\right)}
$$

where

$$
\begin{aligned}
E_{z}(\zeta) & =(v)_{k} e_{I}(\zeta)(1-\langle\zeta, z\rangle)^{-v-k}, & & e_{I}(\zeta)=\zeta_{i_{1}} \cdots \zeta_{i_{k}}, \\
E_{w}(\zeta) & =(v)_{s-k} e_{J}(\zeta)(1-\langle\zeta, w\rangle)^{-v+s-k}, & & e_{J}(\zeta)=\zeta_{j_{1}} \cdots \zeta_{j_{s-k}},
\end{aligned}
$$

it follows that

$$
\left(\partial_{z}^{k} \partial_{w}^{s-k} G\right)(0,0)=(v)_{k}(v)_{s-k} \sum_{|I|=k,|J|=s-k} \overline{A\left(e_{I}, e_{J}\right)} d z_{I} \otimes d w_{J} .
$$

Since $A$ is bounded then

$$
\left\|\left(\partial_{z}^{k} \partial_{w}^{s-k} G\right)(0,0)\right\| \leq C\|A\| .
$$

Let $z \in \mathrm{B}$ and define a bilinear form $A_{z}$ on $L_{a}^{2}\left(d \iota_{v}\right)$ such that

$$
A_{z}(f, g)=A\left(\pi_{v}\left(\varphi_{z}\right) f, \pi_{v}\left(\varphi_{z}\right) g\right),
$$

where $\varphi_{z}$ is the linear fractional mapping (8) and $\pi_{v}$ is the action (13). Then it holds that $\left\|A_{z}\right\|=\|A\|$ and by the same transformation property as in Lemma 3.2, see also [15], it follows that $\tilde{\mathscr{T}}_{s}\left(A_{z}\right)=\pi_{v, s}\left(\varphi_{z}\right) \tilde{\mathscr{T}}_{s}(A)$. Hence, replacing $A$ by $A_{z}$ in (45) yields

$$
\left\|\tilde{\mathscr{T}}_{s}\left(A_{z}\right)(0)\right\| \leq C\left\|A_{z}\right\|=C\|A\|
$$

and

$$
\tilde{\mathscr{T}}_{s}\left(A_{z}\right)(0)=\left(\pi_{v, s}\left(\varphi_{z}\right) \tilde{\mathscr{T}}_{s}(A)\right)(0)=\otimes^{s} \varphi_{z}^{\prime}(0)^{t} \tilde{\mathscr{T}}_{s}(A)(z) J_{\varphi_{z}}(0)^{2 v /(d+1)}
$$

so that

$$
\left\|\otimes^{s} B^{t}(z, z)^{1 / 2} \tilde{\mathscr{T}}_{s}(A)(z)\left(1-|z|^{2}\right)^{v}\right\| \leq C\|A\| .
$$

This proves the lemma.

Lemma 8.5. Let $\tilde{\mathscr{T}}_{s}$ be defined on $\mathscr{T}_{2}$ as in (44). Then $\tilde{\mathscr{T}}_{s}: \mathscr{T}_{2} \rightarrow \mathscr{H}_{v, s}^{2}$ is bounded.

Proof. By Theorem 6.6 it follows that $\sigma: \mathscr{H}_{v, s}^{2} \rightarrow \mathscr{S}_{2}, \sigma(F)=H_{F}^{s}$ defines an isometry. Thus $\sigma^{*}: \mathscr{S}_{2} \rightarrow \mathscr{H}_{v, s}^{2}$ is a partial isometry and therefore bounded. We claim that $\sigma^{*}=\tilde{\mathscr{T}}_{s}$, which actually follows by an identification. Indeed 
let $A$ be a bilinear form of finite rank. We shall prove that $\sigma^{*}(A)=\tilde{\mathscr{T}}_{s}(A)$, which gives the general case. Let $H_{F}^{s}$ be a Hilbert-Schmidt Hankel form. Then

$$
\left\langle H_{F}^{s}, A\right\rangle_{\mathscr{S}_{2}}=\sum_{i, j=1}^{N} H_{F}^{s}\left(e_{i}, e_{j}\right) \overline{A\left(e_{i}, e_{j}\right)}
$$

where $\left\{e_{i}\right\}_{i=1}^{N}$ is an orthonormal set in $\mathscr{H}_{v, s}^{2}$. Since

$$
H_{F}^{s}\left(e_{i}, e_{j}\right)=\int_{\mathrm{B}}\left\langle\otimes^{s} B^{t}(z, z) \mathscr{T}_{s}\left(e_{i}, e_{j}\right)(z), F(z)\right\rangle d \iota_{2 v}(z)
$$

then

$$
\begin{aligned}
\sum_{i, j=1}^{N} H_{F}^{s}\left(e_{i}, e_{j}\right) & \overline{A\left(e_{i}, e_{j}\right)} \\
= & \int_{\mathrm{B}}\left\langle\otimes^{s} B^{t}(z, z) \sum_{i, j=1}^{N} \mathscr{T}_{s}\left(e_{i}, e_{j}\right)(z) \overline{A\left(e_{i}, e_{j}\right)}, F(z)\right\rangle d \iota_{2 v}(z) .
\end{aligned}
$$

On the other hand

$$
\left\langle H_{F}^{s}, A\right\rangle_{\mathscr{L}_{2}}=\langle\sigma(F), A\rangle_{\mathscr{S}_{2}}=\left\langle\sigma^{*}(A), F\right\rangle_{v, s, 2} .
$$

Thus, it remains to prove that

$$
\tilde{\mathscr{T}}_{s}(A)(z)=\sum_{i, j=1}^{N} \mathscr{T}_{s}\left(e_{i}, e_{j}\right)(z) \overline{A\left(e_{i}, e_{j}\right)} .
$$

Since $A(f, g)=0$ if $f$ or $g$ is in $\operatorname{span}\left\{e_{1}, \ldots, e_{N}\right\}^{\perp}$ and since $\left\{\bar{e}_{i} \otimes \bar{e}_{j}\right\}$ is an orthonormal set in $\mathscr{S}_{2}$, where $\bar{e}_{i} \otimes \bar{e}_{j}(f, g)=\left\langle f, e_{i}\right\rangle_{\nu}\left\langle g, e_{j}\right\rangle_{v}$, then

$$
A=\sum_{i, j=1}^{N}\left\langle A, \bar{e}_{i} \otimes \bar{e}_{j}\right\rangle_{\mathscr{S}_{2}} \bar{e}_{i} \otimes \bar{e}_{j}=\sum_{i, j=1}^{N} A\left(e_{i}, e_{j}\right) \bar{e}_{i} \otimes \bar{e}_{j} .
$$

Hence

$$
G(z, w)=\overline{A\left(K_{z}, K_{w}\right)}=\sum_{i, j=1}^{N} \overline{A\left(e_{i}, e_{j}\right)} e_{i}(z) e_{j}(w)
$$

so that

$$
\tilde{\mathscr{T}}_{s}(A)(z)=\left(\mathscr{T}_{s} G\right)(z, z)=\sum_{i, j=1}^{N} \overline{A\left(e_{i}, e_{j}\right)} \mathscr{T}_{s}\left(e_{i}, e_{j}\right)(z)
$$


which proves (46).

Lemma 8.6. Let $\tilde{\mathscr{T}}_{s}$ be defined on $\mathscr{S}_{p}$ as in (44), $2<p<\infty$. Then $\tilde{\mathscr{T}}_{s}: \mathscr{S}_{p} \rightarrow \mathscr{H}_{v, s}^{p}$ is bounded and $\tilde{\mathscr{T}}_{s}\left(H_{F}^{s}\right)=F$ if $H_{F}^{s} \in \mathscr{S}_{p}$.

Proof. It follows from Lemma 8.4, Lemma 8.5 and Riesz-Thorin's interpolation theorem that $\tilde{\mathscr{T}}_{s}: \mathscr{S}_{p} \rightarrow \mathscr{H}_{\nu, s}^{p}$ is bounded for $2<p<\infty$. Also, $\tilde{\mathscr{T}}_{s}\left(H_{F}^{s}\right)=F$ if $H_{F}^{s} \in \mathscr{S}_{2}$. Now define $F_{r}(z)=F(r z)$ for $0<r<1$. Then $H_{F_{r}}^{s} \in \mathscr{T}_{2}$ so that $\tilde{\mathscr{T}}_{s}\left(H_{F_{r}}^{s}\right)=F_{r}$. Since $H_{F}^{s}$ is compact then $F_{r} \rightarrow F$ in $\mathscr{H}_{v, s}^{\infty}$, by the necessity of Theorem 1.1(b) and the proof of Lemma 6.2. On one hand $F_{r} \rightarrow F$ pointwise. On the other hand, by Theorem 1.1(a) and Lemma 8.4, it follows that $\tilde{\mathscr{T}}\left(H_{F_{r}}^{s}\right) \rightarrow \tilde{\mathscr{T}}\left(H_{F}^{s}\right)$. Thus $\tilde{\mathscr{T}}_{s}\left(H_{F}^{s}\right)=F$ if $H_{F}^{s} \in \mathscr{S}_{p}$.

\section{REFERENCES}

1. Bergh, J., and Löfström, J., Interpolation Spaces. An Introduction, Grundlehren Math. Wiss. 223 (1976).

2. Bröcker, T., and Dieck, T., Representations on Compact Lie Groups, Springer-Verlag, 1985.

3. Faraut, J., and Koranyi, A., Analysis on Symmetric Cones, Clarendon Press, 1994.

4. Fergusson, S., and Rochberg, R., Higher order Hilbert-Schmidt Hankel forms and tensors of analytic kernels, Math. Scand. 96 (2005), no. 1, 117-146.

5. Hedenmalm, H., Korenblum, B., Zhu, K., Theory of Bergman Spaces, Springer-Verlag, New York-Berlin-Heidelberg, 2000.

6. Hwang, S., Liu, Y., and Zhang, G., Hilbert spaces of tensor-valued holomorphic functions on the unit ball of $\mathrm{C}^{n}$, Pacific J. Math, 214 (2004), no. 2, 303-322.

7. Janson, S., and Peetre, J., A new generalization of Hankel operators (the case of higher weights), Math. Nachr. 132 (1987), 313-328.

8. Janson, S., Peetre, J., and Rochberg, R., Hankel forms and the Fock space, Rev. Mat. Iberoamericana 3 (1986), 61-138.

9. Knapp, A., Representation Theory of Semisimple Groups, Princeton University Press, Princeton, New Jersey (1986).

10. Loos, O., Bounded symmetric domains and Jordan pairs, The University of California at Irvine, 1977.

11. Peetre, J., Hankel kernels of higher weight for the ball, Nagoya Math. J. 130 (1993), 183-192.

12. Peetre, J., Hankel forms of arbitrary weight over a symmetric domain via the Transvectant, Rocky Mountain J. Math. 24 (1994), no. 3, 1065-1085.

13. Peller, V. V., Hankel Operators and their Applications, Springer-Verlag, 2002.

14. Peller, V. V., Vectorial Hankel operators, commutators and related operators of the Schattenvon Neumann class $\gamma_{p}$, Integral Equations Operator Theory 5 (1982), no. 2, 244-272.

15. Peng L., and Zhang, G., Tensor product of holomorphic representations and bilinear differential operators, J. Funct. Anal. 210 (2004), no. 1, 171-192.

16. Reed, M., and Simon, B., Methods of Modern Mathematical Physics I: Functional Analysis, Academic press, 1980.

17. Rochberg, R., A Kronecker theorem for higher order Hankel forms, Proc. Amer. Math. Soc. 123 (1995), no. 10, 3113-3118.

18. Rochberg, R., Trace ideal criteria for Hankel operators and commutators, Indiana Univ. Math. J. 31 (1982), no. 6, 913-925. 
19. Rosengren, H., Multilinear Hankel forms of higher order and orthogonal polynomials, Math. Scand. 82 (1998), 53-88.

20. Rudin, W., Function Theory in the Unit Ball of $\mathrm{C}^{n}$, Springer-Verlag, 1980.

21. Simon, B., Trace Ideals and their Applications, Cambridge University Press, CambridgeLondon-New York-Melbourne, 1979.

22. Zhu, K., Operator Theory in Function Spaces, Marcel Dekker, Inc., New York, 1990.

DEPARTMENT OF MATHEMATICS

CHALMERS UNIVERSITY OF TECHNOLOGY AND GÖTEBORG UNIVERSITY

SE-412 96 GÖTEBORG

SWEDEN

E-mail: sundhall@math.chalmers.se 